\title{
SHRIMP U-Pb zircon dating of a metagabbro and eclogites from western Dabieshan (Hong'an Block), China, and its tectonic implications
}

\author{
Xiaochun Liu, ${ }^{\mathrm{a}, *}$, Bor-ming Jahn ${ }^{\mathrm{b}}$, Dunyi Liu ${ }^{\mathrm{c}}$, Shuwen Dong ${ }^{\mathrm{a}}$, Sanzhong $\mathrm{Li}^{\mathrm{d}}$ \\ anstitute of Geomechanics, Chinese Academy of Geological Sciences, Beijing 100081, China \\ ${ }^{\mathrm{b}}$ Department of Geosciences, National Taiwan University, Taipei 10699, Taiwan \\ ${ }^{\mathrm{c}}$ Institute of Geology, Chinese Academy of Geological Sciences, Beijing 100026, China \\ ${ }^{\mathrm{d}}$ College of Marine Geosciences, Ocean University of China, Qingdao 266003, China
}

Received 19 February 2004; accepted 20 August 2004

Available online 28 October 2004

\begin{abstract}
In order to better understand the collision tectonics between the Sino-Korean and Yangtze cratons, we performed ion microprobe (SHRIMP) dating on zircons of a metagabbro and four eclogites from the high-pressure/ultrahigh-pressure (HP/ UHP) metamorphic terrane of western Dabieshan (also known as the Hong'an Block), China. New zircon U-Pb data of eclogites from the Huwan and Hong'an HP units suggest that their protoliths were formed during the Neoproterozoic, from $733 \pm 10$ to $684 \pm 15 \mathrm{Ma}$, whereas the metamorphic ages are $229 \pm 12 \mathrm{Ma}$ for an eclogite of the Huwan HP unit, and $213 \pm 5 \mathrm{Ma}$ for that of the Xinxian UHP unit. The age data indicate that western Dabieshan is an integral part of the Dabie-Sulu Triassic HP/ UHP metamorphic belt. A few eclogites with Carboniferous or older zircon ages reported in the literature are limited in the northwestern corner of western Dabieshan. They should not be considered as representative of the whole Huwan unit, and the geological meaning of their occurrence still remains obscure. The identical ages of eclogites from the Huwan and Hong'an units, together with their comparable P-T conditions and similar country rocks, suggest that the two units belong to the same HP slice overlying the UHP slice (=main part of the Xinxian unit). Zircons from a metagabbro in the Balifan tectonic mélange unit located to the north of the HP/UHP complexes yield a Neoproterozoic emplacement age at $582 \pm 11 \mathrm{Ma}$. Taking into account the Yangtze signature for the adjacent Nanwan flysch unit, we infer that the Balifan and Nanwan units represent the basement and cover accretionary complexes, respectively. They were sliced from the Yangtze craton during its northerly subduction. Accordingly, the northern boundary fault of the Nanwan unit should be the Triassic suture between the Sino-Korean and Yangtze cratons. The cooling ages obtained from different units suggest diachronous exhumation of the subducted slab during Triassic time, from ca. $240 \mathrm{Ma}$ for the hanging wall accretionary complex to ca. $210 \mathrm{Ma}$ for the footwall UHP slice. This
\end{abstract}

* Corresponding author. Tel.: +861068 486756; fax: +861068 422326.

E-mail address: liuxchqw@yahoo.com.cn (X. Liu). 
scenario could be explained by a successive subduction of underlying slices with a nearly concomitant exhumation of overlying slices.

(C) 2004 Elsevier B.V. All rights reserved.

Keywords: Zircon dating; Metagabbro; Eclogite; HP/UHP metamorphism; Dabieshan

\section{Introduction}

The Qinling-Dabie-Sulu orogen marks the suture zone between the Sino-Korean and Yangtze cratons. The high-pressure (HP) and ultrahigh-pressure (UHP) metamorphic rocks in this orogen extend from eastern Qinling, through Tongbaishan and Dabieshan, across the Tanlu fault, and then northeastwards to the Sulu region (Fig. 1). Published age data established that the HP and UHP metamorphism took place during the Triassic collision between the two Precambrian cratons (Ames et al., 1993, 1996; Li et al., 1993, 1994, 2000; Eide et al., 1994; Chavagnac and Jahn, 1996; Rowley et al., 1997; Maruyama et al., 1998; Hacker et al., 1998, 2000; Chavagnac et al., 2001; Ayers et al., 2002; Jahn et al., 2003a; Yang et al., 2003). However, based on the isotopic dating of eclogites from the northwestern corner of western Dabieshan, some investigators argued that the HP eclogites in the northern eclogite zone (so-called Huwan shear zone) could have formed in a separate period during the early or late Paleozoic subduction of an oceanic plate beneath the Sino-Korean craton (Jian et al., 1997, 2000; Xu et al., 2000; Li et al., 2001; Sun et al., 2002). This interpretation is at variance with the commonly accepted view that the general structure of Dabieshan is a huge antiform or dome, with HP eclogites occurring in two limbs (Hacker et al., 1998, 2000; Faure et al., 1999, 2003; Eide and Liou, 2000). Indeed, the nature and age of the northern HP eclogite zone and its relation to other HP/UHP eclogites have become a highly controversial issue (Fu et al., 2002; Gao et al., 2002; Jahn and Liu, 2002; Jahn et al., 2003b; Ratschbacher et al., 2003). In this study, we performed a new round of zircon dating on a metagabbro and four eclogites from the major units of western Dabieshan using the SHRIMP technique. The purposes of this work are: (1) to determine the ages of the eclogite protoliths and the HP/UHP metamorphism in the whole western Dabieshan; (2) to examine whether the entire northern eclogite zone was formed in early or late Paleozoic as claimed by some workers; and (3) to present a working model for the tectonic evolution of this terrane.

\section{Geological setting and previous geochronological work}

Western Dabieshan, often called as the Hong'an Block, is located in between Tongbaishan and eastern Dabieshan. It is bounded by the Dawu fault in the west and the Shangma fault in the east (Fig. 1). In this block, various HP and UHP metamorphic rocks and structures are well preserved and the Cretaceous tectonic and thermal overprint is generally weak or absent (Liou et al., 1996; Hacker et al., 1996, 2000; Webb et al., 1999, 2001; Eide and Liou, 2000). Based on the petrological characteristics, six major lithotectonic units can be distinguished (Zhong et al., 1999, 2001; Liu et al., 2004). They are, from north to south, the Nanwan flysch unit, the Balifan tectonic mélange unit, the Huwan HP unit, the Xinxian UHP unit, the Hong'an HP unit and the Mulanshan blueschistgreenschist unit (Fig. 1). The Nanwan flysch unit is composed of Devonian bedded quartzose sandstone, pelite and minor greywacke; they are metamorphosed at the greenschist to epidote-amphibolite facies. The Balifan tectonic mélange unit consists of augen mylonite, mylonitized quartzofeldspathic schist and epidote amphibolite with many large metagabbro blocks. No eclogite has been found. The Huwan HP unit comprises augen gneiss, mylonite, quartzofeldspathic schist, graphite schist, marble and abundant eclogite enclaves. The Xinxian UHP unit is a structural dome composed of granitic to granodioritic gneiss and minor supracrustal rock with many eclogite lenses or pods. Coesite, a diagnostic UHP mineral, has been identified in kyanite-bearing eclogites (Zhang and Liou, 1994; Cui and Wang, 1995; Liu et al., 2004). However, eclogites in the northern and southern marginal zones of this unit only show a HP 


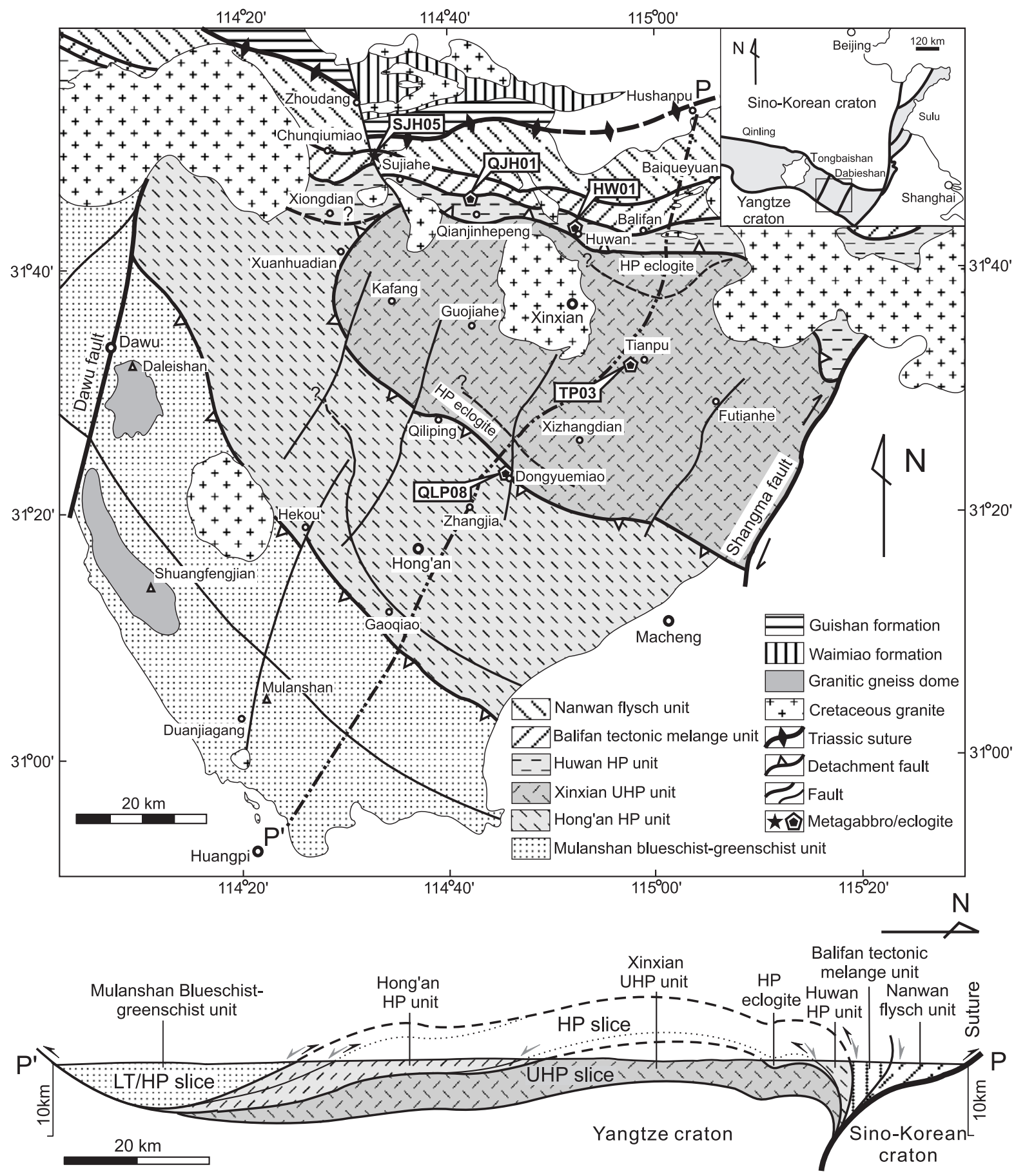

Fig. 1. Simplified geological map of western Dabieshan (also known as the Hong'an Block) with inset showing its location in the QinlingDabie-Sulu orogen of China. P-P' shows a generalized profile across western Dabieshan (modified after Liu et al., 2004). 
paragenesis (Liu et al., 2004). The Hong'an HP unit consists of quartzofeldspathic schist, granitic gneiss and minor graphite schist, quartzite and marble with numerous layered garnet amphibolites as well as eclogite and ultramafic blocks or lenses. The Mulanshan blueschist-greenschist unit mainly comprises bimodal volcanics and some metasedimentary rocks. The above lithotectonic units are mostly separated by multilayered detachment zones related to postcollisional extension (Zhong et al., 1999, 2001), but in the northwestern corner, the boundary between the Huwan and Hong'an units is unclear. In addition, two amphibolite facies complexes, the Guishan and Waimiao formations, occur in the northwest of the Nanwan unit. According to Okay et al. (1993) and Ratschbacher et al. (2003), they belong to the Paleozoic magmatic arc sequence accreted onto the Sino-Korean craton.

In contrast to the well-dated eastern Dabieshan and Sulu region, the geochronological studies in western Dabieshan are scarce. Eide et al. (1994) reported six phengite ${ }^{40} \mathrm{Ar} /{ }^{39} \mathrm{Ar}$ ages of 195 to $230 \mathrm{Ma}$, hence demonstrating that western Dabieshan is a part of the Triassic HP/UHP metamorphic belt. Subsequently, a large number of phengite ${ }^{40} \mathrm{Ar} /{ }^{39} \mathrm{Ar}$ dates obtained by Webb et al. $(1999,2001)$ in the whole area supported this conclusion. Earlier, U-Pb zircon geochronology was mainly performed on eclogites from the northwestern corner of the Huwan unit, where a wide range of metamorphic ages from Silurian to Triassic were obtained (Jian et al., 1997, 2000; Li et al., 2001; Sun et al., 2002; Gao et al., 2002). Jian et al. (1997) reported TIMS U-Pb zircon ages of $400 \pm 2$ and $302 \pm 2 \mathrm{Ma}$ for the Xiongdian eclogite, which are consistent with the later SHRIMP zircon analyses (424 \pm 5 and $301 \pm 13$ Ma, respectively; Jian et al., 2000). These authors interpreted the ca. $400 \mathrm{Ma}$ as the minimum age for the eclogite facies metamorphism and the $300 \mathrm{Ma}$ as the time of retrograde metamorphism. The phengite ${ }^{40} \mathrm{Ar} /{ }^{39} \mathrm{Ar}$ ages of $350-430$ Ma obtained by $\mathrm{Xu}$ et al. (2000) from the same eclogite were intriguingly explained as the retrograde metamorphic age. More recently, using SHRIMP U$\mathrm{Pb}$ dating, Sun et al. (2002) obtained a Carboniferous metamorphic age of $309 \pm 3$ Ma for the zircon rims of three eclogite samples from the Xiongdian and adjacent Hujiawan localities. The age was interpreted as the time of an HP metamorphic event. In addition, ages of 350-440 Ma were also obtained for zircon cores from the same samples. These ages were regarded as the time of igneous intrusion or lowpressure metamorphism in the southern margin of the Sino-Korean craton. These authors also reported a protolith age of $752 \pm 17 \mathrm{Ma}$ and a Triassic metamorphic age of $232 \pm 10 \mathrm{Ma}$ for an eclogite from Xuanhuadian, about $5 \mathrm{~km}$ south of Xiongdian. The above age data have led these authors to infer that two sutures, a Carboniferous and a Triassic, existed on the two sides of the Huwan unit. Sun et al. (2002) insisted that the Carboniferous eclogites were not affected by the Triassic eclogite facies event, but Gao et al. (2002) reported a Triassic SHRIMP age of $216 \pm 4$ Ma for six zircons from precisely the same Xiongdian eclogite. Moreover, a protolith age of $631 \pm 3$ Ma was also obtained for the Hujiawan eclogite by TIMS dating (Li et al., 2001). It is clear that, even using the same SHRIMP or TIMS U-Pb dating technique on the same eclogite, the results have not always been consistent.

\section{Petrological characterization and $P-T$ conditions of the dated rocks}

To obtain the geochronological information of the whole subducted complexes in western Dabieshan, five well-preserved metabasic rocks were chosen for SHRIMP U-Pb zircon dating. They are the Sujiahe metagabbro (sample SJH05) from the Balifan tectonic mélange unit, the Qianjinhepeng (sample QJH01) and Huwan (sample HW01) eclogites from the Huwan HP unit, the Tianpu eclogite (sample TP03) from the Xinxian UHP unit and the Dongyuemiao eclogite (sample QLP08) from the Hong'an HP unit. Their localities are shown in Fig. 1, and their mineral assemblages and peak metamorphic conditions are presented in Table 1.

The Sujiahe metagabbro is representative of the numerous large metagabbro blocks in the Balifan unit. It occurs as a $70 \times 100 \mathrm{~m}^{2}$ body enclosed in granitic mylonite. The body is cut by several 2-m-wide finegrained gneissic metagabbro veins, which are very common in the western part of the Balifan unit (i.e., Dingyuan formation; Li et al., 2001). Sample SJH05 shows a typical gabbroic texture, with mineral grains $3-8 \mathrm{~mm}$ in size. The original igneous phases are 
Table 1

Sampling localities, mineral assemblages and peak metamorphic P-T conditions of the dated rocks from western Dabieshan

\begin{tabular}{|c|c|c|c|c|c|c|}
\hline \multirow[t]{2}{*}{ Sample } & \multirow[t]{2}{*}{ Location } & \multirow[t]{2}{*}{ Coordinates } & \multirow[t]{2}{*}{ Lithology } & \multirow[t]{2}{*}{ Mineral assemblage } & \multicolumn{2}{|c|}{ Peak P-T estimates } \\
\hline & & & & & $\mathrm{T}\left({ }^{\circ} \mathrm{C}\right)$ & $\mathrm{P}$ (kbar) \\
\hline SJH05 & Sujiahe & $\begin{array}{l}\text { N } 31^{\circ} 49.08^{\prime} \\
\text { E } 114^{\circ} 32.93^{\prime}\end{array}$ & Metagabbro & $\mathrm{Cpx}+\mathrm{Pl}+\mathrm{Amp}+\mathrm{Zo}+\mathrm{Bt}+\mathrm{Mag}$ & & \\
\hline QJH01 & Qianjinhepeng & $\begin{array}{l}\text { N } 31^{\circ} 45.96^{\prime} \\
\text { E } 114^{\circ} 40.67^{\prime}\end{array}$ & Eclogite & $\mathrm{Grt}+\mathrm{Cpx}+\mathrm{Amp}+\mathrm{Ep}+\mathrm{Qtz}+\mathrm{Rt}$ & $610 \pm 40$ & $>12$ \\
\hline HW01 & Huwan & $\begin{array}{l}\text { N } 31^{\circ} 43.63^{\prime} \\
\text { E } 114^{\circ} 52.57^{\prime}\end{array}$ & Eclogite & $\mathrm{Grt}+\mathrm{Cpx}+\mathrm{Amp}+\mathrm{Phg}+\mathrm{Ep}+\mathrm{Qtz}+\mathrm{Rt}+\mathrm{Ttn}$ & $570 \pm 30$ & $>12$ \\
\hline ТР03 & Tianpu & $\begin{array}{l}\text { N } 31^{\circ} 31.96^{\prime} \\
\text { E } 114^{\circ} 57.28^{\prime}\end{array}$ & Eclogite & $\mathrm{Grt}+\mathrm{Cpx}+\mathrm{Ky}+\mathrm{Gln}+\mathrm{Phg}+\mathrm{Ep}+\mathrm{Pg}+\mathrm{Cs} / \mathrm{Qtz}+\mathrm{Rt}$ & $640 \pm 30$ & $29 \pm 3$ \\
\hline QLP08 & Dongyuemiao & $\begin{array}{l}\text { N } 31^{\circ} 23.65^{\prime} \\
\text { E } 114^{\circ} 45.77^{\prime}\end{array}$ & Eclogite & $\mathrm{Grt}+\mathrm{Cpx}+\mathrm{Amp}+\mathrm{Phg}+\mathrm{Ep}+\mathrm{Pg}+\mathrm{Qtz}+\mathrm{Rt}$ & $530 \pm 30$ & $20 \pm 2$ \\
\hline
\end{tabular}

Mineral abbreviations: Amp=amphibole; Bt=biotite; $\mathrm{Cpx}=$ clinopyroxene; $\mathrm{Cs}=$ coesite; $\mathrm{Ep}=$ epidote; Gln=glaucophane; Grt=garnet; Ky=kyanite; Mag=magnetite; Phg=phengite; $\mathrm{Pg}=$ paragonite; $\mathrm{Pl}=$ plagioclase; $\mathrm{Qtz}=$ quartz; Rt=rutile; Ttn=titanite; $\mathrm{Zo}=\mathrm{zoisite}$.

plagioclase, clinopyroxene and magnetite. Plagioclase is mostly transformed to fine-grained zoisite+albite, but its primary crystal form and polysynthetic twins are often preserved. The rims of clinopyroxene are completely replaced by amphiboles. Amphiboles also occur as fine-grained aggregates in matrix. Magnetite grains show abundant fractures, which are commonly filled with fine-grained biotites. These features indicate that the gabbro underwent an epidote-amphibolite facies metamorphism.

The Qianjinhepeng eclogite occurs as an elongated lens, about $300 \mathrm{~m}$ in width, in quartzofeldspathic gneiss in the northern margin of the Huwan unit. The eclogite is strongly deformed and mostly retrograded to amphibolite. Sample QJH01 comprises garnet, clinopyroxene, amphibole, rutile and minor epidote and quartz. Garnet is euhedral and coarse-grained, with grain sizes of $1-3 \mathrm{~mm}$. It commonly contains numerous inclusions of amphibole, epidote and, occasionally, clinopyroxene. Clinopyroxene is finegrained $(\leq 1 \mathrm{~mm})$ and strongly oriented. Most amphiboles crystallized simultaneously with other minerals, but some occurring around garnet porphyroblast were formed at the retrograde stage.

The Huwan eclogite block $\left(>100 \times 100 \mathrm{~m}^{2}\right)$ occurs in augen mylonite in the southern margin of the Huwan unit. The block was strongly deformed and overprinted by retrograde epidote-amphibolite facies metamorphism. Sample HW01 was collected from the weakly deformed core of the block. It is composed predominantly of garnet, clinopyroxene, amphibole, quartz, rutile and a trace amount of phengite and epidote. Large garnet crystals often contain inclusions of amphibole and a small amount of clinopyroxene, epidote and quartz. Coronas around clinopyroxene are made up of small garnet grains. Phengite and epidotes only occur along the weakly deformed plane, suggesting a late growth.

The Tianpu eclogite occurs as a lenticular body $(\sim 10 \mathrm{~m}$ wide) in granitic gneiss in the Xinxian unit. It is characterized by the presence of coesite, hence suggesting UHP metamorphism (Zhang and Liou, 1994). Sample TP03 consists of garnet, clinopyroxene, kyanite, glaucophane, phengite, epidote, quartz, rutile and minor paragonite. All garnet crystals are fine-grained $(\leq 1 \mathrm{~mm})$ and sometimes contain inclusions of amphibole, epidote, quartz and rutile. A quartz pseudomorph after coesite was identified in an annular garnet grain. Clinopyroxene and epidote are generally coarser than garnet. Glaucophane in the matrix mainly occurs as large porphyroblasts, up to 3 $\mathrm{mm}$ in length. It commonly contains inclusions of garnet, kyanite, epidote and quartz. It is also in sharp contact with garnet, omphacite and kyanite, suggesting that it formed during a late stage of UHP recrystallization (Zhang and Liou, 1994; Eide and Liou, 2000). Some reworked glaucophane rims are found in contact with paragonite, indicating a thermal overprinting below the stability of kyanite.

The Dongyuemiao eclogite occurs as a lens of $5 \times 25 \mathrm{~m}^{2}$ in quartzofeldspathic schist on the northern border of the Hong'an unit. The rock shows nearly equigranular texture with grain sizes about $0.4 \mathrm{~mm}$. Sample QLP08 is composed dominantly of garnet, 
clinopyroxene, amphibole and epidote, and a subordinate amount of phengite, paragonite, quartz and rutile. Garnet is often annular and envelops phengite, amphibole, epidote and/or quartz. Clinopyroxene exhibits optical zoning, as does clinopyroxene from all other eclogites in western Dabieshan. Amphibole is in textural equilibrium with other minerals.

Electron microprobe analyses show that both garnet and clinopyroxene from the above four eclogites exhibit chemical zoning (for details, see Liu et al., 2004). In garnet, $\mathrm{Mg}$ and $\mathrm{Fe}^{2+}$ increase while $\mathrm{Mn}$ and $\mathrm{Ca}$ decrease from core to rim. In clinopyroxene, the zoning shows an increase of $\mathrm{Al}$ and $\mathrm{Ca}$ and a decrease of $\mathrm{Fe}^{3+}$ from core to rim. The zoning pattern indicates that the peak temperature and pressure have been roughly attained in their rims. Based on a garnet-clinopyroxene thermometry (Powell, 1985), jadeite-albite-quartz and garnetclinopyroxene-phengite barometry (Carswell and Harley, 1990; Carswell et al., 1997) and THERMOCALC program (Powell et al., 1998), the peak P-T conditions are estimated to be $610 \pm 40{ }^{\circ} \mathrm{C}$ and $>12$ kbar for the Qianjinhepeng eclogite, $570 \pm 30{ }^{\circ} \mathrm{C}$ and $>12$ kbar for the Huwan eclogite, $640 \pm 30{ }^{\circ} \mathrm{C}$ and $29 \pm 3 \mathrm{kbar}$ for the Tianpu eclogite, and $530 \pm 30{ }^{\circ} \mathrm{C}$ and $20 \pm 2 \mathrm{kbar}$ for the Dongyuemiao eclogite (see also Liu et al., 2004). This suggests that the peak metamorphic temperature and pressure of eclogites in western Dabieshan are lower by 50 to $150{ }^{\circ} \mathrm{C}$ than those in the eastern Dabieshan and Sulu UHP terranes (Liou et al., 2002).

Note that the temperature range $\left(640-530{ }^{\circ} \mathrm{C}\right)$ is roughly at the threshold of the blocking temperatures for two important chronometers involved in study of UHP metamorphic rocks: garnet $\mathrm{Sm}-\mathrm{Nd}$ (blocking temperature $T_{\mathrm{B}}=850-650{ }^{\circ} \mathrm{C}$; e.g., Mezger et al., 1992; Burton et al., 1995; Zhou and Hensen, 1995; Günther and Jagoutz, 1997; Thöni, 2002) and white mica $\mathrm{Rb}-\mathrm{Sr}$ (phengite or muscovite, $T_{\mathrm{B}}=500 \pm 50{ }^{\circ} \mathrm{C}$; Jaeger et al., 1967). In fact, the clear chemical zoning of garnet crystals is clear evidence for nonequilibration. In this case, trace element and isotopic equilibrium is probably not achieved. Our Nd-Sr isotope study indicates that the $\mathrm{Sm}-\mathrm{Nd}$ and several $\mathrm{Rb}-\mathrm{Sr}$ isotope systems in eclogites of western Dabieshan have not been equilibrated during the metamorphism (Jahn and Liu, 2002; Jahn et al., 2003b). By contrast, zircon crystallization may take place in a very large temperature range. In HP and UHP metamorphic terranes, zircon may grow during the prograde as well as retrograde recrystallization (Tilton et al., 1991; Gebauer et al., 1997; Rubatto et al., 1999; Thöni, 2002). Metamorphic recrystallization of zircon and resetting of its $\mathrm{U}-\mathrm{Pb}$ systematics could be enhanced by fluids even at moderate temperatures $\left(<600{ }^{\circ} \mathrm{C}\right.$; Rubatto et al., 1999; Liati and Gebauer, 1999). In this paper, we mainly report the results of zircon $\mathrm{U}-\mathrm{Pb}$ dating, supplemented by a few $\mathrm{Rb}-\mathrm{Sr}$ phengite analyses. The problem of $\mathrm{Sm}-\mathrm{Nd}$ and oxygen isotope disequilibrium will be discussed in another paper in preparation.

\section{Analytical procedures}

Zircons were extracted from the five samples by crushing, sieving, heavy liquid and hand picking at the Geological Survey of Hebei Province, China. Zircon grains were mounted in an epoxy disc along with the Temara zircon standard (417 Ma; Black et al., 2003) and polished down to expose their centers. Internal structures of zircon grains were revealed by cathodoluminescence (CL) image carried out on a CAMEBAX SX51 electron microprobe at the Institute of Geology and Geophysics, Chinese Academy of Sciences. The operating conditions were $15 \mathrm{kV}$ accelerating voltage and a $20-\mathrm{nA}$ beam current. Metamorphic mineral inclusions in zircon were identified using backscattered electron (BSE) image and electron microprobe analysis. $\mathrm{SiO}_{2}$ phases (quartz and coesite) were detected by a RANISOW RW-1000 Laser Raman microspectroscope at Peking University. The selection of zircon domains for SHRIMP analyses was dictated by the CL and BSE images.

Zircon was analyzed for $\mathrm{U}-\mathrm{Th}-\mathrm{Pb}$ isotopes using the SHRIMP II sensitive high-resolution ion microprobe at the Institute of Geology, Chinese Academy of Gelological Sciences. Instrumental conditions and data acquisition procedures were similar to those described by Williams and Claesson (1987), Compston et al. (1992) and Liu et al. (2003a). A primary ion beam of $4.5 \mathrm{nA}, 10 \mathrm{kV} \mathrm{O}_{2}^{-}$and $25-30 \mu \mathrm{m}$ spot were used. A mass resolution was ca. 5400 at $1 \%$ peak height. The data were collected in sets of five scans throughout the $\mathrm{Zr}, \mathrm{Pb}, \mathrm{U}$ and $\mathrm{Th}$ isotope species and a reference zircon was analyzed every two or three 
Table 2

SHRIMP U-Pb analyses of zircons for a metagabbro and eclogites from western Dabieshan

\begin{tabular}{|c|c|c|c|c|c|c|c|c|c|}
\hline \multirow[t]{2}{*}{ Spot } & \multirow{2}{*}{$\begin{array}{l}\mathrm{U} \\
(\mathrm{ppm})\end{array}$} & \multirow{2}{*}{$\begin{array}{l}\text { Th } \\
\text { (ppm) }\end{array}$} & \multirow{2}{*}{\multicolumn{2}{|c|}{$\begin{aligned} \mathrm{Th} / \mathrm{U} & \mathrm{Pb}^{*} \\
& (\mathrm{ppm})\end{aligned}$}} & \multirow{2}{*}{$\begin{array}{l}\text { Common } \\
{ }^{206} \mathrm{~Pb}(\%)\end{array}$} & \multicolumn{2}{|l|}{ Isotopic ratios } & Ages (Ma) & \multirow[t]{2}{*}{ CL domain } \\
\hline & & & & & & ${ }^{206} \mathrm{~Pb} /{ }^{238} \mathrm{U} \quad{ }^{207} \mathrm{~Pb} /{ }^{235} \mathrm{U}$ & ${ }^{207} \mathrm{~Pb} /{ }^{206} \mathrm{~Pb}$ & $\overline{{ }^{206} \mathrm{~Pb} /{ }^{238} \mathrm{U} \quad{ }^{207} \mathrm{~Pb} /{ }^{206} \mathrm{~Pb}}$ & \\
\hline
\end{tabular}

\begin{tabular}{lrrlrl}
\hline \multicolumn{6}{l}{ Sujiahe metagabbro $($ SJH05) } \\
1.1 & 77 & 39 & 0.50 & 6 & 0.01 \\
2.1 & 87 & 91 & 1.05 & 9 & 0.01 \\
3.1 & 957 & 1401 & 1.46 & 121 & 0.00 \\
4.1 & 50 & 36 & 0.73 & 4 & 0.03 \\
5.1 & 79 & 77 & 0.97 & 9 & 0.01 \\
6.1 & 65 & 44 & 0.67 & 7 & 0.01 \\
7.1 & 95 & 39 & 0.41 & 8 & 0.02 \\
7.2 & 33 & 20 & 0.60 & 3 & 0.02 \\
8.1 & 74 & 47 & 0.63 & 8 & 0.00 \\
9.1 & 42 & 24 & 0.58 & 5 & 0.02 \\
10.1 & 53 & 40 & 0.76 & 6 & 0.01 \\
11.1 & 60 & 53 & 0.89 & 6 & 0.01 \\
12.1 & 50 & 33 & 0.65 & 5 & 0.00 \\
13.1 & 117 & 68 & 0.58 & 12 & 0.00 \\
14.1 & 58 & 42 & 0.73 & 6 & 0.00 \\
15.1 & 71 & 67 & 0.95 & 8 & 0.00 \\
16.1 & 36 & 18 & 0.49 & 4 & 0.00 \\
17.1 & 187 & 137 & 0.73 & 18 & 0.00 \\
18.1 & 68 & 57 & 0.83 & 7 & 0.00 \\
19.1 & 62 & 48 & 0.77 & 6 & 0.01 \\
20.1 & 75 & 50 & 0.67 & 7 & 0.01 \\
21.1 & 54 & 32 & 0.59 & 5 & 0.00 \\
22.1 & 41 & 25 & 0.62 & 4 & 0.01 \\
23.1 & 69 & 47 & 0.68 & 7 & 0.04
\end{tabular}

$\begin{array}{llll}0.0770 \pm 32 & 0.594 \pm 41 & 0.0560 \pm 29 & 478 \pm 19 \\ 0.0911 \pm 71 & 0.734 \pm 66 & 0.0584 \pm 21 & 562 \pm 42 \\ 0.0838 \pm 55 & 0.696 \pm 47 & 0.0608 \pm 5 & 518 \pm 33 \\ 0.0706 \pm 80 & 0.541 \pm 111 & 0.0557 \pm 87 & 440 \pm 48 \\ 0.0971 \pm 41 & 0.754 \pm 55 & 0.0563 \pm 30 & 597 \pm 24 \\ 0.0997 \pm 41 & 0.783 \pm 53 & 0.0569 \pm 29 & 613 \pm 24 \\ 0.0876 \pm 38 & 0.577 \pm 53 & 0.0478 \pm 36 & 541 \pm 22 \\ 0.0932 \pm 52 & 0.536 \pm 76 & 0.0418 \pm 52 & 574 \pm 30 \\ 0.1011 \pm 41 & 0.785 \pm 49 & 0.0563 \pm 24 & 621 \pm 24 \\ 0.1043 \pm 44 & 0.710 \pm 87 & 0.0494 \pm 55 & 639 \pm 26 \\ 0.0939 \pm 40 & 0.767 \pm 44 & 0.0593 \pm 20 & 579 \pm 24 \\ 0.0933 \pm 38 & 0.726 \pm 44 & 0.0564 \pm 23 & 575 \pm 22 \\ 0.1001 \pm 43 & 0.807 \pm 47 & 0.0585 \pm 19 & 615 \pm 25 \\ 0.0990 \pm 42 & 0.802 \pm 40 & 0.0587 \pm 13 & 609 \pm 25 \\ 0.1014 \pm 46 & 0.835 \pm 48 & 0.0597 \pm 18 & 623 \pm 27 \\ 0.0964 \pm 39 & 0.806 \pm 38 & 0.0606 \pm 12 & 593 \pm 23 \\ 0.0976 \pm 45 & 0.817 \pm 51 & 0.0607 \pm 22 & 600 \pm 26 \\ 0.0855 \pm 78 & 0.706 \pm 68 & 0.0599 \pm 13 & 529 \pm 46 \\ 0.0939 \pm 46 & 0.791 \pm 51 & 0.0611 \pm 23 & 578 \pm 27 \\ 0.0819 \pm 35 & 0.640 \pm 41 & 0.0567 \pm 25 & 507 \pm 21 \\ 0.0817 \pm 108 & 0.595 \pm 104 & 0.0529 \pm 52 & 506 \pm 65 \\ 0.0873 \pm 48 & 0.691 \pm 56 & 0.0574 \pm 30 & 540 \pm 29 \\ 0.0982 \pm 45 & 0.766 \pm 57 & 0.0566 \pm 30 & 604 \pm 26 \\ 0.0925 \pm 42 & 0.823 \pm 249 & 0.0645 \pm 189 & 570 \pm 25\end{array}$

Qianjinhepeng eclogite (QJH01)

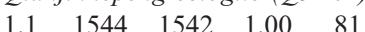

$\begin{array}{rrrrrr}2.1 & 819 & 688 & 0.84 & 88 & 0.00 \\ 3.1 & 241 & 197 & 0.82 & 39 & 0.01 \\ 4.1 & 142 & 360 & 2.54 & 23 & 0.01 \\ 5.1 & 139 & 142 & 1.02 & 18 & 0.01 \\ 6.1 & 784 & 736 & 0.94 & 99 & 0.01 \\ 7.1 & 372 & 351 & 0.94 & 33 & 0.01 \\ 8.1 & 140 & 166 & 1.18 & 19 & 0.01 \\ 9.1 & 3132 & 783 & 0.37 & 74 & 0.01 \\ 10.1 & 1174 & 263 & 0.22 & 48 & 0.00 \\ 11.1 & 823 & 248 & 0.30 & 57 & 0.00 \\ 12.1 & 1115 & 716 & 0.64 & 99 & 0.00 \\ 13.1 & 1560 & 1419 & 0.91 & 85 & 0.01 \\ 14.1 & 649 & 1532 & 2.36 & 33 & 0.02 \\ 15.1 & 1535 & 2050 & 1.34 & 110 & 0.00 \\ 16.1 & 1272 & 302 & 0.24 & 53 & 0.01 \\ 17.1 & 2452 & 1416 & 0.58 & 109 & 0.01 \\ 18.1 & 666 & 891 & 1.34 & 28 & 0.04 \\ 19.1 & 750 & 1291 & 1.72 & 55 & 0.01\end{array}$

Huwan eclogite (HW01)

\begin{tabular}{|c|c|c|c|}
\hline 534 & 1008 & 1.89 & 97 \\
\hline 476 & 695 & 1.46 & 83 \\
\hline 910 & 1518 & 1.67 & 162 \\
\hline
\end{tabular}

$\begin{array}{llll}0.0438 \pm 28 & 0.320 \pm 28 & 0.0531 \pm 28 & 276 \pm 17 \\ 0.0976 \pm 41 & 0.787 \pm 36 & 0.0585 \pm 8 & 600 \pm 24 \\ 0.1557 \pm 44 & 1.353 \pm 76 & 0.0630 \pm 28 & 933 \pm 25 \\ 0.1188 \pm 69 & 1.061 \pm 91 & 0.0648 \pm 36 & 724 \pm 40 \\ 0.1217 \pm 42 & 1.033 \pm 101 & 0.0616 \pm 54 & 740 \pm 24 \\ 0.1126 \pm 43 & 0.930 \pm 44 & 0.0599 \pm 14 & 688 \pm 25 \\ 0.0787 \pm 35 & 0.606 \pm 57 & 0.0559 \pm 43 & 488 \pm 21 \\ 0.1170 \pm 48 & 0.948 \pm 75 & 0.0588 \pm 37 & 713 \pm 28 \\ 0.0362 \pm 19 & 0.207 \pm 16 & 0.0415 \pm 22 & 229 \pm 12 \\ 0.0430 \pm 24 & 0.272 \pm 20 & 0.0459 \pm 18 & 271 \pm 15 \\ 0.0713 \pm 26 & 0.547 \pm 32 & 0.0557 \pm 23 & 444 \pm 16 \\ 0.0864 \pm 36 & 0.620 \pm 40 & 0.0521 \pm 23 & 534 \pm 22 \\ 0.0500 \pm 28 & 0.338 \pm 24 & 0.0491 \pm 18 & 315 \pm 17 \\ 0.0405 \pm 32 & 0.224 \pm 27 & 0.0400 \pm 34 & 256 \pm 20 \\ 0.0615 \pm 44 & 0.455 \pm 35 & 0.0537 \pm 10 & 385 \pm 27 \\ 0.0438 \pm 18 & 0.300 \pm 24 & 0.0498 \pm 31 & 276 \pm 11 \\ 0.0441 \pm 24 & 0.270 \pm 20 & 0.0444 \pm 19 & 278 \pm 15 \\ 0.0381 \pm 24 & 0.208 \pm 34 & 0.0396 \pm 57 & 241 \pm 15 \\ 0.0797 \pm 32 & 0.585 \pm 48 & 0.0532 \pm 36 & 495 \pm 19\end{array}$

$452 \pm 118$ Homogeneous bright rim $546 \pm 82$ Homogeneous bright core $613 \pm 18$ Dark core

$438 \pm 393$ Homogeneous bright rim $464 \pm 123$ Homogeneous bright core $489 \pm 114$ Weak oscillatory core $89 \pm 171$ Oscillatory core Homogeneous bright rim

$465 \pm 98$ Weak oscillatory core $165 \pm 240$ Homogeneous bright rim $577 \pm 75 \quad$ Homogeneous bright core $469 \pm 91$ Weak oscillatory core $548 \pm 74$ Homogeneous bright rim $557 \pm 49$ Weak oscillatory core $593 \pm 66$ Homogeneous bright core $626 \pm 44$ Homogeneous bright rim $630 \pm 80$ Homogeneous bright rim $600 \pm 47$ Oscillatory rim $644 \pm 82$ Weak oscillatory rim $480 \pm 99$ Weak oscillatory core $322 \pm 239$ Weak oscillatory rim $506 \pm 121$ Homogeneous bright core $476 \pm 122$ Homogeneous bright rim $757 \pm 757$ Homogeneous bright rim

$\begin{array}{ll}331 \pm 123 & \text { Cloudy core } \\ 548 \pm 30 & \text { Oscillatory core } \\ 709 \pm 99 & \text { Fine-scale oscillatory core } \\ 766 \pm 123 & \text { Oscillatory and sector core } \\ 659 \pm 200 & \text { Oscillatory core } \\ 601 \pm 51 & \text { Oscillatory rim } \\ 448 \pm 181 & \text { Oscillatory rim } \\ 560 \pm 144 & \text { Oscillatory core } \\ & \text { Cloudy core } \\ & \text { Cloudy core } \\ 439 \pm 96 & \text { Mixed core } \\ 288 \pm 105 & \text { Mixed rim } \\ 151 \pm 88 & \text { Cloudy rim } \\ & \text { Cloudy core } \\ 357 \pm 44 & \text { Mixed core } \\ 183 \pm 151 & \text { Cloudy core } \\ & \text { Cloudy core } \\ & \text { Cloudy core } \\ 337 \pm 159 & \text { Mixed core }\end{array}$

$\begin{array}{llllll}0.1281 \pm 64 & 1.125 \pm 59 & 0.0637 \pm 7 & 777 \pm 37 & 731 \pm 23 & \text { Oscillatory core } \\ 0.1335 \pm 56 & 1.142 \pm 54 & 0.0620 \pm 10 & 808 \pm 32 & 675 \pm 34 & \text { Oscillatory core } \\ 0.1307 \pm 50 & 1.148 \pm 46 & 0.0637 \pm 5 & 792 \pm 29 & 732 \pm 16 & \text { Oscillatory rim }\end{array}$


Table 2 (continued)

\begin{tabular}{|c|c|c|c|c|c|c|c|c|c|c|c|}
\hline \multirow{2}{*}{ Spot } & \multirow{2}{*}{$\begin{array}{l}\mathrm{U} \\
(\mathrm{ppm})\end{array}$} & \multirow{2}{*}{$\begin{array}{l}\text { Th } \\
\text { (ppm) }\end{array}$} & \multirow[t]{2}{*}{$\mathrm{Th} / \mathrm{U}$} & \multirow{2}{*}{$\begin{array}{l}\mathrm{Pb}^{*} \\
\text { (ppm) }\end{array}$} & \multirow{2}{*}{$\begin{array}{l}\text { Common } \\
{ }^{206} \mathrm{~Pb}(\%)\end{array}$} & \multicolumn{3}{|c|}{ Isotopic ratios } & \multicolumn{2}{|l|}{ Ages (Ma) } & \multirow[t]{2}{*}{ CL domain } \\
\hline & & & & & & ${ }^{206} \mathrm{~Pb} /{ }^{238} \mathrm{U}$ & ${ }^{207} \mathrm{~Pb} /{ }^{235} \mathrm{U}$ & ${ }^{207} \mathrm{~Pb} /{ }^{206} \mathrm{~Pb}$ & ${ }^{206} \mathrm{~Pb} /{ }^{238} \mathrm{U}$ & ${ }^{207} \mathrm{~Pb} /{ }^{206} \mathrm{~Pb}$ & \\
\hline \multicolumn{12}{|c|}{ Huwan eclogite (HW01) } \\
\hline 3.1 & 527 & 1190 & 2.26 & 94 & 0.00 & $0.1184 \pm 107$ & $1.039 \pm 96$ & $0.0636 \pm 8$ & $722 \pm 62$ & $729 \pm 26$ & Oscillatory core \\
\hline 4.1 & 2017 & 6394 & 3.17 & 417 & 0.00 & $0.1194 \pm 70$ & $1.042 \pm 62$ & $0.0633 \pm 4$ & $727 \pm 41$ & $719 \pm 13$ & Homogeneous dark core \\
\hline 5.1 & 316 & 513 & 1.63 & 55 & 0.00 & $0.1293 \pm 47$ & $1.108 \pm 48$ & $0.0622 \pm 11$ & $784 \pm 27$ & $680 \pm 40$ & Oscillatory core \\
\hline 6.1 & 1292 & 3240 & 2.51 & 252 & 0.00 & $0.1251 \pm 74$ & $1.098 \pm 67$ & $0.0637 \pm 7$ & $760 \pm 42$ & $730 \pm 24$ & Weak oscillatory rim \\
\hline 7.1 & 569 & 1485 & 2.61 & 107 & 0.00 & $0.1167 \pm 69$ & $1.010 \pm 62$ & $0.0628 \pm 7$ & $712 \pm 40$ & $700 \pm 25$ & Oscillatory rim \\
\hline 8.1 & 540 & 1235 & 2.29 & 111 & 0.00 & $0.1360 \pm 58$ & $1.191 \pm 57$ & $0.0636 \pm 11$ & $822 \pm 33$ & $727 \pm 37$ & Oscillatory core \\
\hline 9.1 & 505 & 886 & 1.76 & 76 & 0.00 & $0.1096 \pm 65$ & $0.981 \pm 66$ & $0.0649 \pm 17$ & $670 \pm 38$ & $773 \pm 56$ & Oscillatory core \\
\hline 10.1 & 443 & 698 & 1.58 & 70 & 0.00 & $0.1183 \pm 51$ & $1.014 \pm 47$ & $0.0622 \pm 8$ & $721 \pm 30$ & $681 \pm 26$ & Oscillatory core \\
\hline 10.2 & 473 & 813 & 1.72 & 84 & 0.00 & $0.1298 \pm 61$ & $1.132 \pm 57$ & $0.0632 \pm 8$ & $787 \pm 35$ & $716 \pm 28$ & Oscillatory rim \\
\hline 11.1 & 385 & 507 & 1.32 & 68 & 0.00 & $0.1399 \pm 52$ & $1.238 \pm 49$ & $0.0642 \pm 7$ & $844 \pm 29$ & $747 \pm 22$ & Weak oscillatory rim \\
\hline 12.1 & 673 & 1531 & 2.27 & 132 & 0.00 & $0.1294 \pm 50$ & $1.143 \pm 46$ & $0.0641 \pm 6$ & $785 \pm 28$ & $744 \pm 20$ & Oscillatory core \\
\hline 13.1 & 2005 & 6202 & 3.09 & 443 & 0.00 & $0.1286 \pm 65$ & $1.141 \pm 59$ & $0.0643 \pm 4$ & $780 \pm 37$ & $752 \pm 12$ & Homogeneous dark core \\
\hline 14.1 & 1740 & 5951 & 3.42 & 377 & 0.00 & $0.1199 \pm 47$ & $1.059 \pm 43$ & $0.0641 \pm 5$ & $730 \pm 27$ & $744 \pm 16$ & Homogeneous dark rim \\
\hline 15.1 & 131 & 218 & 1.66 & 23 & 0.00 & $0.1270 \pm 64$ & $1.144 \pm 63$ & $0.0654 \pm 12$ & $771 \pm 37$ & $786 \pm 38$ & Weak oscillatory rim \\
\hline 16.1 & 428 & 884 & 2.06 & 75 & 0.00 & $0.1199 \pm 43$ & $1.093 \pm 44$ & $0.0661 \pm 10$ & $730 \pm 25$ & $810 \pm 31$ & Oscillatory core \\
\hline 17.1 & 1452 & 4239 & 2.92 & 312 & 0.00 & $0.1285 \pm 47$ & $1.130 \pm 44$ & $0.0638 \pm 6$ & $779 \pm 27$ & $734 \pm 19$ & Oscillatory rim \\
\hline 18.1 & 1180 & 2704 & 2.29 & 223 & 0.00 & $0.1243 \pm 49$ & $1.095 \pm 45$ & $0.0639 \pm 5$ & $755 \pm 28$ & $739 \pm 17$ & Weak oscillatory core \\
\hline
\end{tabular}

Tianpu eclogite (TP03)

$\begin{array}{lrrrrr}1.1 & 157 & 6 & 0.04 & 5 & 0.01 \\ 2.1 & 82 & 4 & 0.04 & 2 & 0.01 \\ 3.1 & 50 & 1 & 0.02 & 2 & 0.03 \\ 4.1 & 72 & 4 & 0.05 & 2 & 0.02 \\ 5.1 & 86 & 1 & 0.01 & 3 & 0.02 \\ 6.1 & 72 & 3 & 0.04 & 2 & 0.02 \\ 7.1 & 87 & 6 & 0.07 & 3 & 0.03 \\ 8.1 & 107 & 4 & 0.04 & 3 & 0.03 \\ 9.1 & 154 & 5 & 0.03 & 5 & 0.01 \\ 10.1 & 156 & 6 & 0.04 & 5 & 0.03 \\ 11.1 & 78 & 2 & 0.03 & 2 & 0.05 \\ 12.1 & 120 & 7 & 0.06 & 4 & 0.02 \\ 13.1 & 137 & 8 & 0.06 & 4 & 0.02\end{array}$

Dongyuemiao eclogite (QLP08)

$\begin{array}{lrrrrlllllll}1.1 & 97 & 108 & 1.12 & 13 & 0.02 & 0.1149 \pm 49 & 0.953 \pm 60 & 0.0602 \pm 25 & 701 \pm 28 & 609 \pm 92 & \text { Oscillatory core } \\ 2.1 & 242 & 431 & 1.78 & 37 & 0.00 & 0.1073 \pm 67 & 0.917 \pm 65 & 0.0620 \pm 16 & 657 \pm 39 & 673 \pm 55 & \text { Oscillatory core } \\ 3.1 & 87 & 145 & 1.67 & 14 & 0.01 & 0.1146 \pm 50 & 0.984 \pm 58 & 0.0623 \pm 21 & 699 \pm 29 & 685 \pm 75 & \text { Oscillatory core } \\ 4.1 & 127 & 171 & 1.35 & 18 & 0.01 & 0.1086 \pm 43 & 0.920 \pm 50 & 0.0614 \pm 20 & 664 \pm 25 & 655 \pm 73 & \text { Oscillatory core } \\ 5.1 & 375 & 236 & 0.63 & 62 & 0.00 & 0.1526 \pm 72 & 1.454 \pm 73 & 0.0691 \pm 8 & 916 \pm 41 & 902 \pm 24 & \text { Fine-scale oscillatory core } \\ 6.1 & 435 & 753 & 1.73 & 68 & 0.00 & 0.1125 \pm 54 & 0.972 \pm 52 & 0.0627 \pm 12 & 687 \pm 31 & 698 \pm 41 & \text { Oscillatory core } \\ 7.1 & 65 & 105 & 1.62 & 10 & 0.02 & 0.1132 \pm 48 & 0.872 \pm 70 & 0.0559 \pm 35 & 691 \pm 28 & 448 \pm 146 & \text { Oscillatory core } \\ 8.1 & 115 & 103 & 0.89 & 15 & 0.01 & 0.1172 \pm 50 & 0.999 \pm 53 & 0.0619 \pm 16 & 714 \pm 29 & 669 \pm 58 & \text { Weak oscillatory core } \\ 9.1 & 660 & 1440 & 2.18 & 112 & 0.00 & 0.1126 \pm 81 & 0.978 \pm 72 & 0.0630 \pm 6 & 688 \pm 47 & 708 \pm 19 & \text { Oscillatory core } \\ 10.1 & 379 & 1297 & 3.43 & 76 & 0.00 & 0.1126 \pm 56 & 0.982 \pm 53 & 0.0632 \pm 11 & 688 \pm 33 & 716 \pm 36 & \text { Oscillatory core } \\ 11.1 & 177 & 189 & 1.07 & 23 & 0.01 & 0.1093 \pm 47 & 0.861 \pm 46 & 0.0572 \pm 15 & 668 \pm 28 & 498 \pm 59 & \text { Oscillatory core } \\ 12.1 & 154 & 158 & 1.02 & 19 & 0.00 & 0.1067 \pm 49 & 0.882 \pm 52 & 0.0600 \pm 19 & 654 \pm 29 & 602 \pm 69 & \text { Weak oscillatory core } \\ 13.1 & 93 & 109 & 1.17 & 13 & 0.00 & 0.1131 \pm 45 & 0.993 \pm 49 & 0.0637 \pm 16 & 690 \pm 26 & 732 \pm 54 & \text { Oscillatory core } \\ 14.1 & 367 & 240 & 0.65 & 148 & 0.00 & 0.3523 \pm 172 & 7.289 \pm 362 & 0.1501 \pm 7 & 1945 \pm 83 & 2347 \pm 8 & \text { Dark oscillatory core } \\ 15.1 & 443 & 300 & 0.68 & 80 & 0.00 & 0.1648 \pm 102 & 1.683 \pm 106 & 0.0741 \pm 5 & 983 \pm 56 & 1044 \pm 13 & \text { Fine-scale oscillatory core }\end{array}$

$\mathrm{Pb} *$ denotes radiogenic $\mathrm{Pb}$. Common ${ }^{206} \mathrm{~Pb}(\%)$ represents the proportion of common ${ }^{206} \mathrm{~Pb}$ in total ${ }^{206} \mathrm{~Pb}$ measured. Common $\mathrm{Pb}$ was corrected using the measured ${ }^{204} \mathrm{~Pb}$ and a model common $\mathrm{Pb}$ composition (Cumming and Richards, 1975). All uncertainties are $1 \sigma$.
Cloudy core
Cloudy core
Cloudy core
Cloudy core
Cloudy core
Cloudy core
Cloudy core
Cloudy core
Cloudy core
Cloudy core
Cloudy core
Cloudy core
Cloudy core

$\begin{array}{llll}0.0338 \pm 14 & 0.208 \pm 21 & 0.0446 \pm 39 & 214 \pm 9 \\ 0.0318 \pm 16 & 0.223 \pm 26 & 0.0509 \pm 50 & 202 \pm 10 \\ 0.0335 \pm 15 & 0.223 \pm 56 & 0.0482 \pm 116 & 213 \pm 10 \\ 0.0331 \pm 15 & 0.200 \pm 44 & 0.0439 \pm 92 & 210 \pm 9 \\ 0.0342 \pm 15 & 0.168 \pm 32 & 0.0356 \pm 65 & 217 \pm 10 \\ 0.0338 \pm 15 & 0.161 \pm 38 & 0.0345 \pm 78 & 214 \pm 9 \\ 0.0344 \pm 19 & 0.189 \pm 37 & 0.0399 \pm 72 & 218 \pm 12 \\ 0.0336 \pm 14 & 0.198 \pm 34 & 0.0428 \pm 69 & 213 \pm 9 \\ 0.0337 \pm 14 & 0.191 \pm 23 & 0.0411 \pm 45 & 214 \pm 8 \\ 0.0341 \pm 13 & 0.152 \pm 21 & 0.0325 \pm 42 & 216 \pm 8 \\ 0.0336 \pm 16 & 0.166 \pm 69 & 0.0359 \pm 147 & 213 \pm 10 \\ 0.0353 \pm 16 & 0.223 \pm 37 & 0.0458 \pm 71 & 223 \pm 10 \\ 0.0321 \pm 16 & 0.190 \pm 20 & 0.0431 \pm 38 & 203 \pm 10\end{array}$


analyses. The measured ${ }^{206} \mathrm{~Pb} /{ }^{238} \mathrm{U}$ ratios were corrected using reference zircon SL13 (572 Ma). The PRAWN data reduction software (Williams et al., 1996) was used for data processing. Ages were calculated using the following constants: $\lambda\left({ }^{238} \mathrm{U}\right)=$ $0.155125 \mathrm{Ga}^{-1}, \lambda\left({ }^{235} \mathrm{U}\right)=0.98485 \mathrm{Ga}^{-1}, \lambda\left({ }^{232} \mathrm{Th}\right)=$ $0.049475 \mathrm{Ga}^{-1}$. Correction for common $\mathrm{Pb}$ was made by the measured ${ }^{204} \mathrm{~Pb}$ and a model common $\mathrm{Pb}$ composition of Cumming and Richards (1975). The age uncertainty given in Table 2 represents one standard deviation $(1 \sigma)$, but the weighted mean ${ }^{206} \mathrm{~Pb} /{ }^{238} \mathrm{U}$ or ${ }^{207} \mathrm{~Pb} /{ }^{206} \mathrm{~Pb}$ ages are quoted at $2 \sigma$ or $95 \%$ confidence level.

\section{Geochronological results}

\subsection{Sujiahe metagabbro (SJH05)}

Zircons from sample SJH05 are $60-200 \mu \mathrm{m}$ in length, subhedral to euhedral and short prismatic, with a length to width ratio of about 1.5. CL images show that most zircon grains are homogeneous and unzoned, but usually contain a dark core (Fig. 2a), which is shown by the bright area in BSE images (Fig. 2b). Some grains have very weak oscillatory zonation. The SHRIMP analysis shows that the dark core (spot 3.1) contains high $\mathrm{U}$ and Th contents (975 and $1401 \mathrm{ppm}$, respectively) and has a $\mathrm{Th} / \mathrm{U}$ ratio of 1.46 , whereas in the homogeneous or oscillatory domain, $\mathrm{U}$ and $\mathrm{Th}$ contents are much lower, varying from 33 to $187 \mathrm{ppm}$ and from 20 to $137 \mathrm{ppm}$, respectively. Their Th/U ratios are also lower, from 0.41 to 1.05 . The $C L$ patterns and $\mathrm{Th} / \mathrm{U}$ ratios are indicative of a magmatic origin (Rubatto and Gebauer, 2000). Twenty-four spot analyses on 23 zircon grains were performed on this sample. Of them, 22 spots yielded a weighted mean ${ }^{206} \mathrm{~Pb} /{ }^{238} \mathrm{U}$ age of $582 \pm 11 \mathrm{Ma}$ (Fig. 3). This is interpreted as the emplacement age of the gabbro. The meaning of two younger ages of $478 \pm 19 \mathrm{Ma}$ (spot 1.1) and $440 \pm 48 \mathrm{Ma}$ (spot 4.1) is unclear; it could be due either to a partial $\mathrm{Pb}$ loss or to the analytical uncertainty.

\subsection{Qianjinhepeng eclogite (QJH01)}

Zircons from sample QJH01 are small, with grain sizes ranging from 30 to $60 \mathrm{~mm}$. Based on morphology, distribution of mineral inclusions and CL image, the zircons could be divided into three groups. Group I zircons are short prismatic, with a length to width ratio of about 1.5. Occasionally, they contain inclusions of apatite, ilmenite and Kfeldspar. In CL images, they exhibit oscillatory zonation (Fig. 2c and d), hence arguing for a magmatic origin. Some grains show a very thin overgrowth rim ( $\leq 5 \mu \mathrm{m}$; Fig. $2 \mathrm{~d})$. Group II zircons are rounded or irregular. They present a weak oscillatory core with a few apatite inclusions and a thick, cloudy overgrowth rim (up to $20 \mu \mathrm{m}$ ) with many inclusions of quartz, amphibole, biotite and apatite (Fig. 2e and f). Group III zircons are also rounded or irregular; but no relict core is present. They are cloudy in CL images (Fig. 2g) and contain numerous inclusions of quartz and minor amount of apatite, amphibole, phengite and rutile (Fig. 2h). These features indicate a metamorphic origin of the Group III zircons.

Nineteen $\mathrm{U}-\mathrm{Pb}$ analyses on 19 zircons reveal a very complex age pattern (Fig. 4). ${ }^{206} \mathrm{~Pb} /{ }^{238} \mathrm{U}$ ages show a very large range from $933 \pm 25$ to $229 \pm 12$ Ma. The oldest age of $933 \pm 25 \mathrm{Ma}$ (spot 3.1) is found in a Group I zircon with very fine scale oscillatory zonation and a $\mathrm{Th} / \mathrm{U}$ ratio of 0.82 . The zircon probably represents an early Neoproterozoic inheritance. A group of four zircons (spots 4.1, 5.1, 6.1 and 8.1) form a concordant population with a weighted mean ${ }^{206} \mathrm{~Pb} /{ }^{238} \mathrm{U}$ age of $716 \pm 28 \mathrm{Ma}$. Their $\mathrm{Th} / \mathrm{U}$ ratios range from 0.94 to 2.54 . This age is thus taken to be the emplacement age of the eclogite protolith. Other Group I zircons show younger ages of $600 \pm 24 \mathrm{Ma}$ (spot 2.1) and $488 \pm 21 \mathrm{Ma}$ (spot 7.1), probably reflecting a $\mathrm{Pb}$ loss during the later metamorphism. Group II zircons give a scattered age pattern ranging from $534 \pm 22$ to $385 \pm 27 \mathrm{Ma}$ (spots $11.1,12.1,15.1$ and 19.1). These ages could be due to the analyses on mixed zones, including oscillatory core and cloudy rim as revealed by CL images. They do not form a unique cluster on the concordia plot (Fig. 4), so they do not seem to reflect any meaningful geological event. The ages of Group III zircons are concentrated between $315 \pm 17$ and $229 \pm 12 \mathrm{Ma}$ (8 analyses). Th/U ratios for these zircons are highly variable from 0.22 to 2.36 . Due to the possible presence of invisible inherited core, we consider the youngest age of $229 \pm 12 \mathrm{Ma}$ (spot 9.1) 

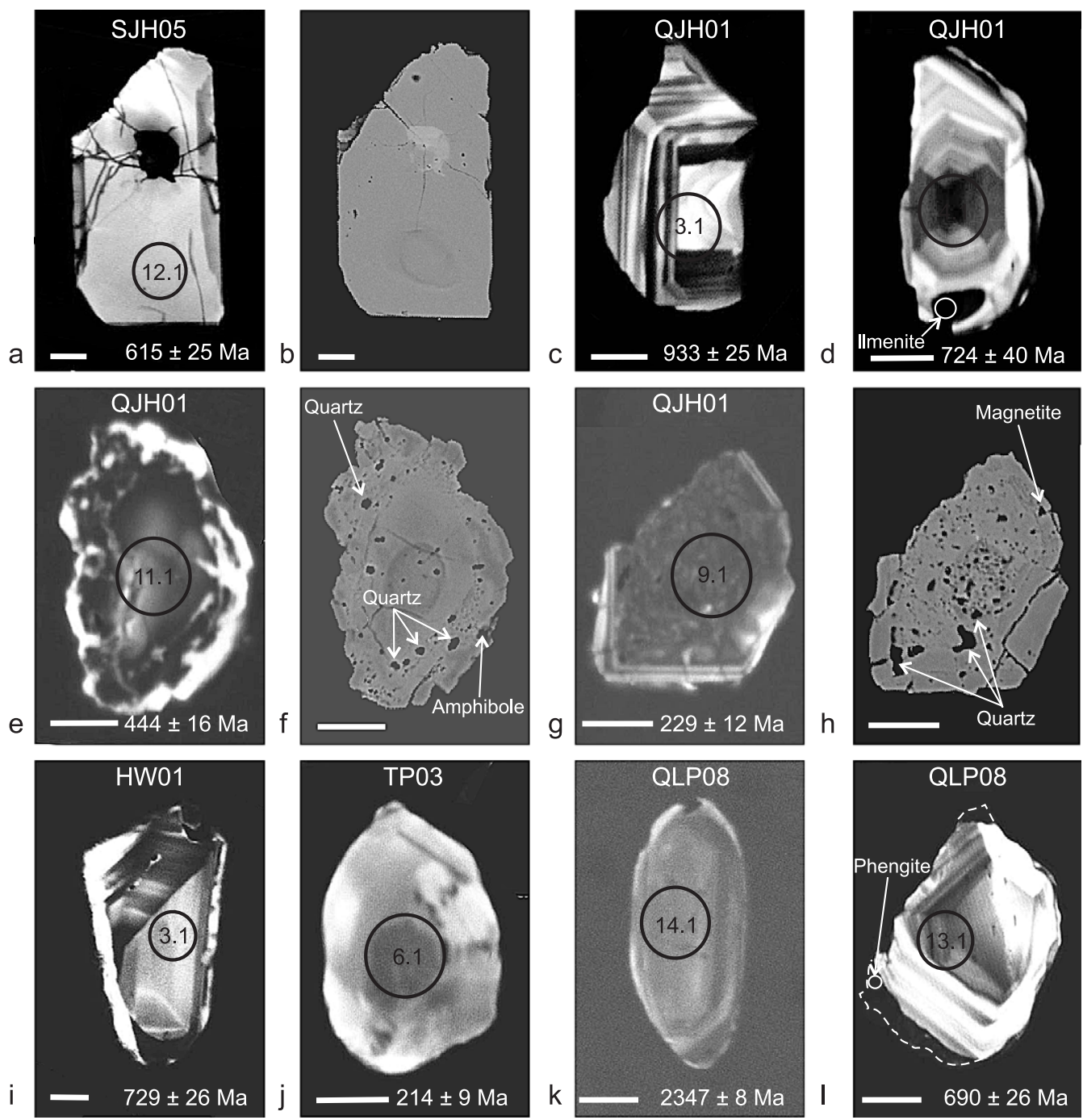

Fig. 2. Cathodoluminescence (CL) and backscattered electron (BSE) images of zircons from a metagabbro and eclogites in western Dabieshan. (a) CL image of a zircon from sample SJH05 showing weak oscillatory zonation with a dark core. (b) BSE image of the same zircon as in (a) showing a bright core. (c) CL image of a zircon from sample QJH01 showing fine-scale oscillatory zonation. (d) CL image of a zircon from sample QJH01 showing oscillatory and sector zonations. A thin overgrowth rim is also present. (e) CL image of a zircon from sample QJH01 showing a weak oscillatory core and a thick cloudy rim. (f) BSE image of the same zircon as in (e) showing an inclusion-poor core and an inclusion-rich rim. (g) CL image of a zircon from sample QJH01 showing cloudy inner domain with an oscillatory rim. (h) BSE image of the same zircon as in (g) showing numerous mineral inclusions. (i) CL image of a zircon from sample HW01 showing oscillatory zonation with a bright overgrowth rim. (j) CL image of a zircon from sample TP03 showing cloudy pattern. (k) CL image of a zircon from sample QLP08 showing dark oscillatory zonation. (1) CL image of a zircon from sample QLP08 showing oscillatory zonation and a dark overgrowth rim. Circles with numbers are SHRIMP analytical spots with their identification numbers. Ages are given at $1 \sigma$ (see Table 2). Scale bars are $20 \mu \mathrm{m}$.

as the maximum age for the HP metamorphic event. Note that the data lying to the left of the concordia, particularly for younger ages, are caused by the high background ${ }^{204} \mathrm{~Pb}$ values, which are suggestive of mineral inclusions in zircons.

\subsection{Huwan eclogite (HW01)}

Zircons from sample HW01 are euhedral and short prismatic, with $50-250 \mu \mathrm{m}$ in length, and have length to width ratios of about 1.5 . Their $\mathrm{CL}$ images show 


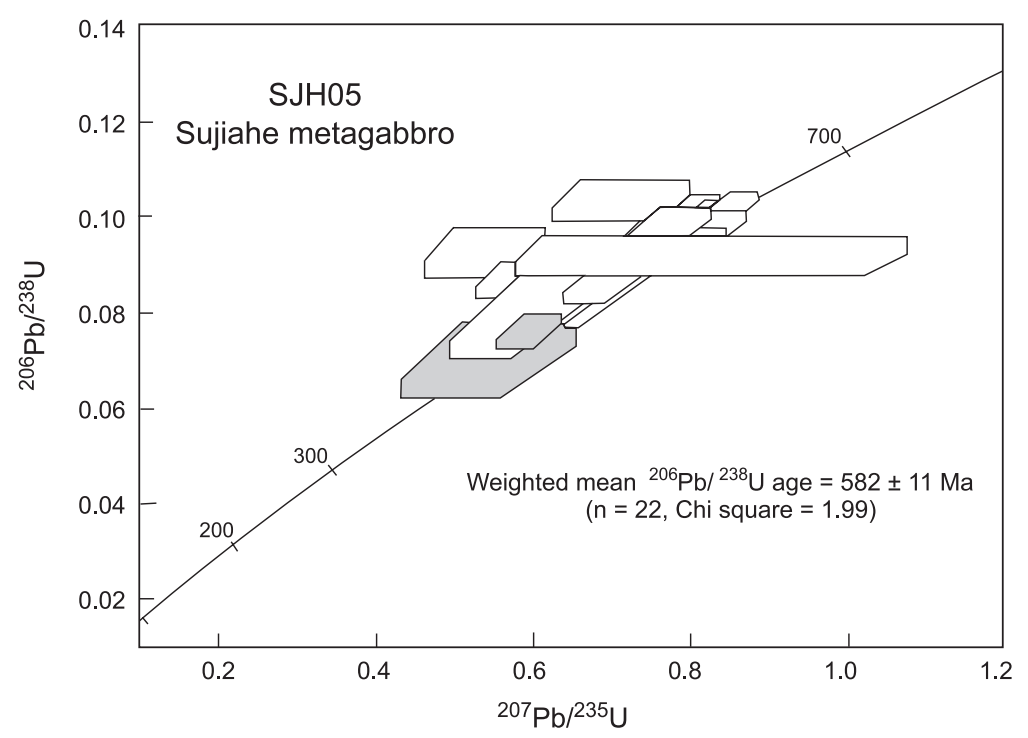

Fig. 3. U-Pb concordia diagram for zircons from the Sujiahe metagabbro (SJH05).

weak oscillatory zonation. Most grains have a bright and small overgrowth rim $(\leq 8 \mu \mathrm{m})$ (Fig. $2 \mathrm{i})$ which is too narrow to be analyzed. Others do not show any metamorphic overgrowth. Twenty spot analyses on 18 zircons plot tightly on or near the concordia (Fig. 5), yielding a weighted mean ${ }^{206} \mathrm{~Pb} /{ }^{238} \mathrm{U}$ age of $766 \pm 14 \mathrm{Ma}$ (Chi square=1.59), which is slightly older than the weighted mean ${ }^{207} \mathrm{~Pb} /{ }^{206} \mathrm{~Pb}$ age of
$733 \pm 10$ Ma. Because zircons in this sample are uranium rich that are reversely discordant, the ${ }^{207} \mathrm{~Pb} /{ }^{206} \mathrm{~Pb}$ age is more meaningful (Wingate et al., 1998; Paulsson and Austrheim, 2003). Their high $\mathrm{Th} / \mathrm{U}$ ratios of 1.32 to 3.42 undoubtedly indicate a magmatic origin. Consequently, the age of ca. 730 Ma represents the protolith emplacement time of the Huwan eclogite.

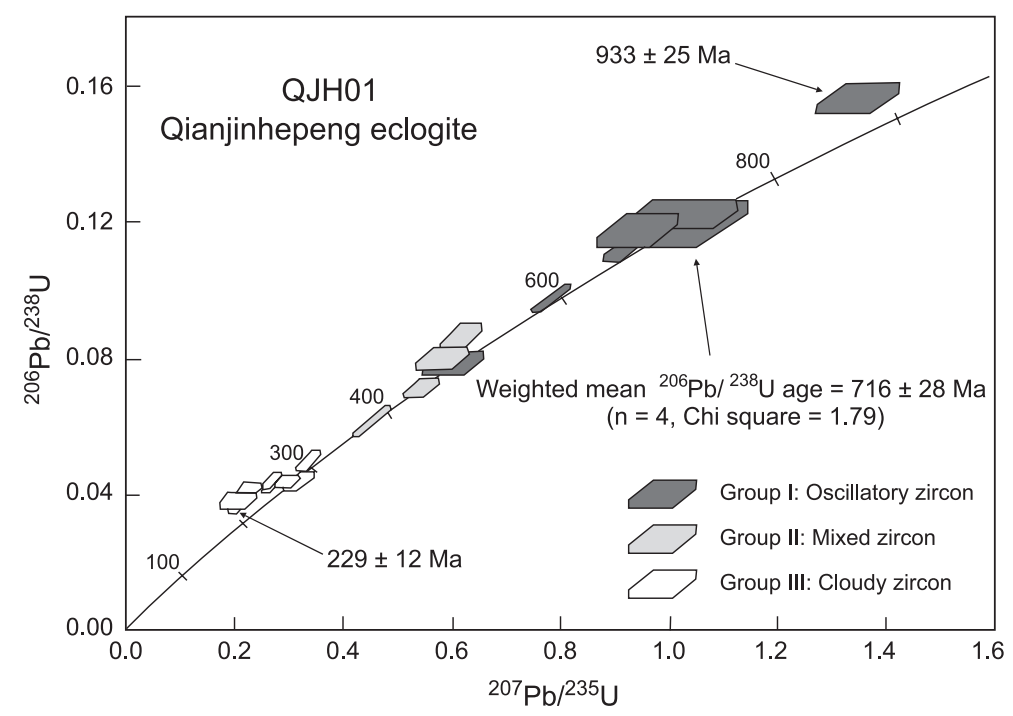

Fig. 4. U-Pb concordia diagram for zircons from the Qianjinhepeng eclogite (QJH01). 


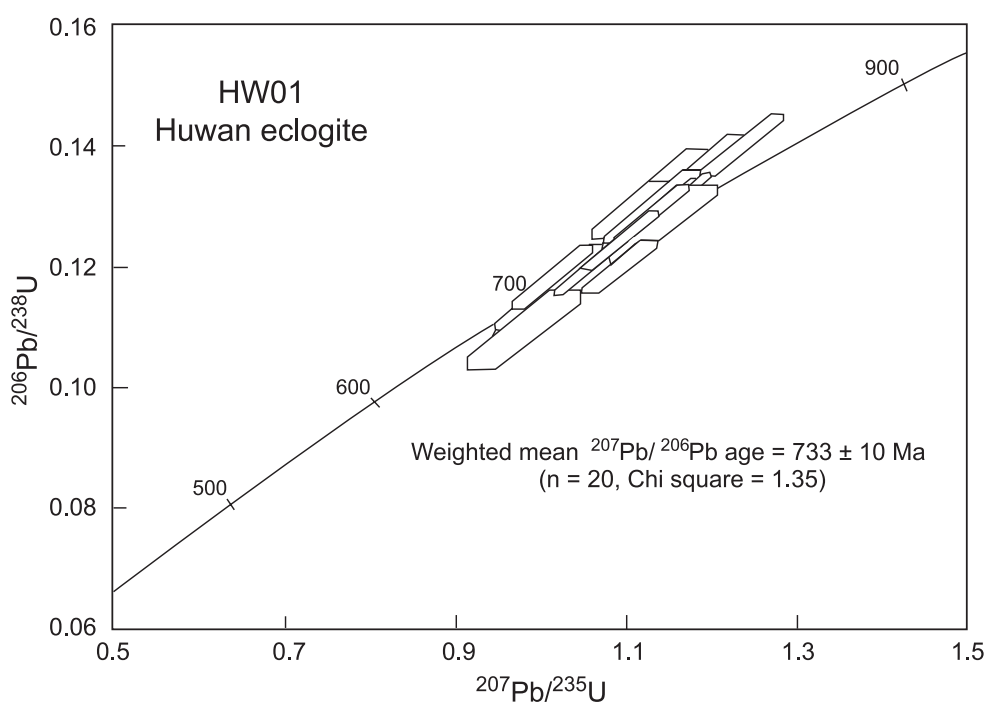

Fig. 5. U-Pb concordia diagram for zircons from the Huwan eclogite (HW01).

\subsection{Tianpu eclogite (TP03)}

Zircons from sample TP03 are rounded and very small, with grain sizes about $20-50 \mathrm{~mm}$. They are cloudy in CL images (Fig. 2j) and sometimes show a narrow bright rim. Thirteen spot analyses on 13 zircons define a weighted mean ${ }^{206} \mathrm{~Pb} /{ }^{238} \mathrm{U}$ age of $213 \pm 5$ Ma (Fig. 6). All zircons have very low Th contents (1-8 ppm) and $\mathrm{Th} / \mathrm{U}$ ratios (only 0.010.07 ), as typically observed in metamorphic zircon domains (e.g., Williams and Claesson, 1987; Gebauer et al., 1997; Rubatto and Gebauer, 2000). The age is straightforward interpreted as the time of the metamorphic recrystallization. Note that no Neoproterozoic protolith ages were obtained from this sample.

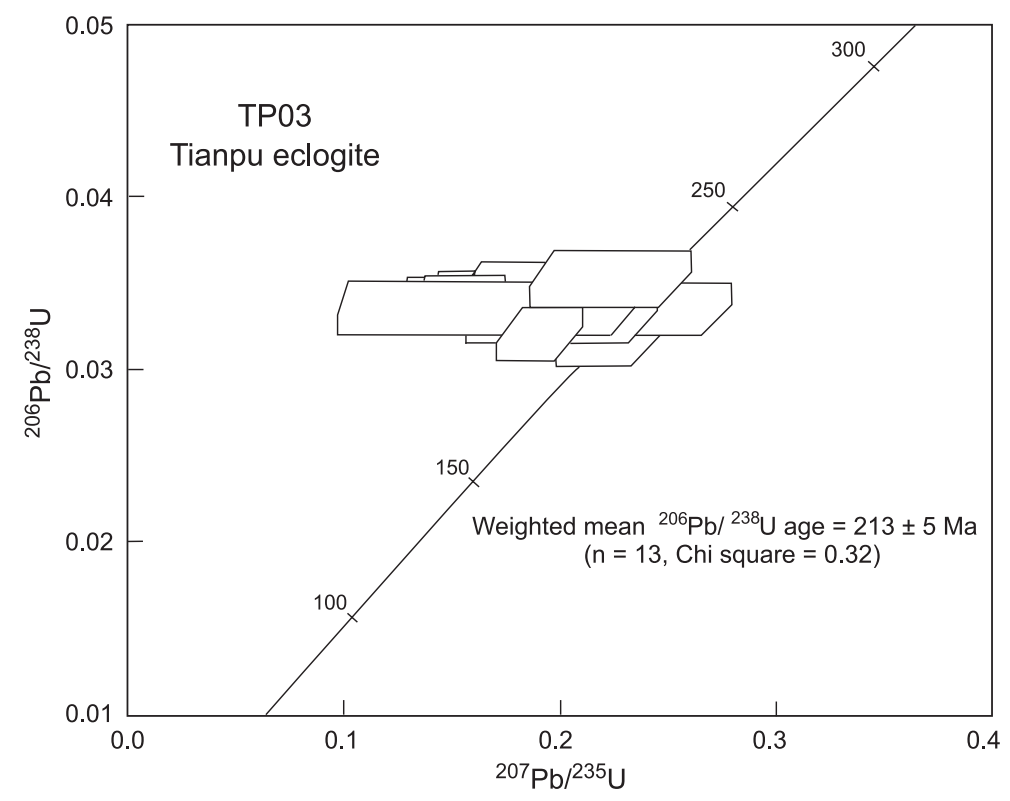

Fig. 6. U-Pb concordia diagram for zircons from the Tianpu eclogite (TP03). 


\subsection{Dongyuemiao eclogite (QLP08)}

Zircons from sample QLP08 are 30-80 $\mu \mathrm{m}$ in length, anhedral to subhedral and short prismatic, with a length to width ratio of about 1.5. Except for a few grains with fine-scale or dark oscillatory zonation in $\mathrm{CL}$ images (Fig. 2k), the zircons are mainly regularly zoned and have an irregular, dark thin rim. The rims are too small for analysis $(\leq 15 \mu \mathrm{m}$; Fig. 21). The occurrence of phengite inclusion in these rims suggests that the overgrowth of zircon was formed during the HP metamorphism. Of $15 \mathrm{U}-\mathrm{Pb}$ analyses, one spot on dark oscillatory zonation (spot 14.1) yields a very old discordant age. Its ${ }^{207} \mathrm{~Pb} /{ }^{206} \mathrm{~Pb}$ age of $2347 \pm 8$ Ma represents a Paleoproterozoic inheritance. Two other grains with fine-scale oscillatory zonation yield Neoproterozoic inherited ${ }^{207} \mathrm{~Pb} /{ }^{206} \mathrm{~Pb}$ ages of $902 \pm 24 \mathrm{Ma}$ (spot 5.1) and $1044 \pm 13 \mathrm{Ma}$ (spot 15.1), similar to the old age obtained for sample QJH01 (spot 3.1). The other 12 zircons with uniformly oscillatory zonation form a concordant cluster giving a weighted mean ${ }^{206} \mathrm{~Pb} /{ }^{238} \mathrm{U}$ age of $684 \pm 15 \mathrm{Ma}$ (Fig. 7), identical to the weighted mean ${ }^{207} \mathrm{~Pb} /{ }^{206} \mathrm{~Pb}$ age of $686 \pm 26 \mathrm{Ma}$ (Chi square=1.66). $\mathrm{Their} \mathrm{Th} / \mathrm{U}$ ratios range from 0.89 to 3.43. It is evident that this Neoproterozoic age represents the protolith age of the eclogite.

\section{Discussion}

\subsection{Ages of eclogite precursor and metamorphism}

Our SHRIMP U-Pb zircon data on three eclogites (samples QJH01, HW01 and QLP08) from western Dabieshan indicate that the protoliths of eclogite were emplaced during the Neoproterozoic, from $733 \pm 10$ to $684 \pm 15 \mathrm{Ma}$. These ages agree with those reported by Ames et al. $(1993,1996)$ from eastern Dabieshan and the Sulu region. They are also comparable with protolith ages of orthogneiss from eastern Dabieshan (Rowley et al., 1997; Xue et al., 1997; Hacker et al., 1998, 2000; Liu et al., 2003b). As Hacker et al. (1998, 2000) suggested, such ages with a distinctive $\sim 750$ Ma signature on various lithologies is in favor of their affiliation to the Yangtze craton. Based on the CL images and $\mathrm{Th} / \mathrm{U}$ ratios, a few zircons from samples QJH01 and QLP08 giving an early Neoproterozoic inherited age of 902-1044 Ma are also of magmatic origin. In fact, zircons of similar ages have also been found in Neoproterozoic granites in the Yangtze craton (Li et al., 2003a,b). They were also interpreted as xenocryst ages. A Paleoproterozoic age of $2347 \pm 8$ Ma obtained for a zircon xenocryst of sample QLP08 is fairly in agreement within error with the upper intercept age of $2458 \pm 76 \mathrm{Ma}$ of a gneiss from the

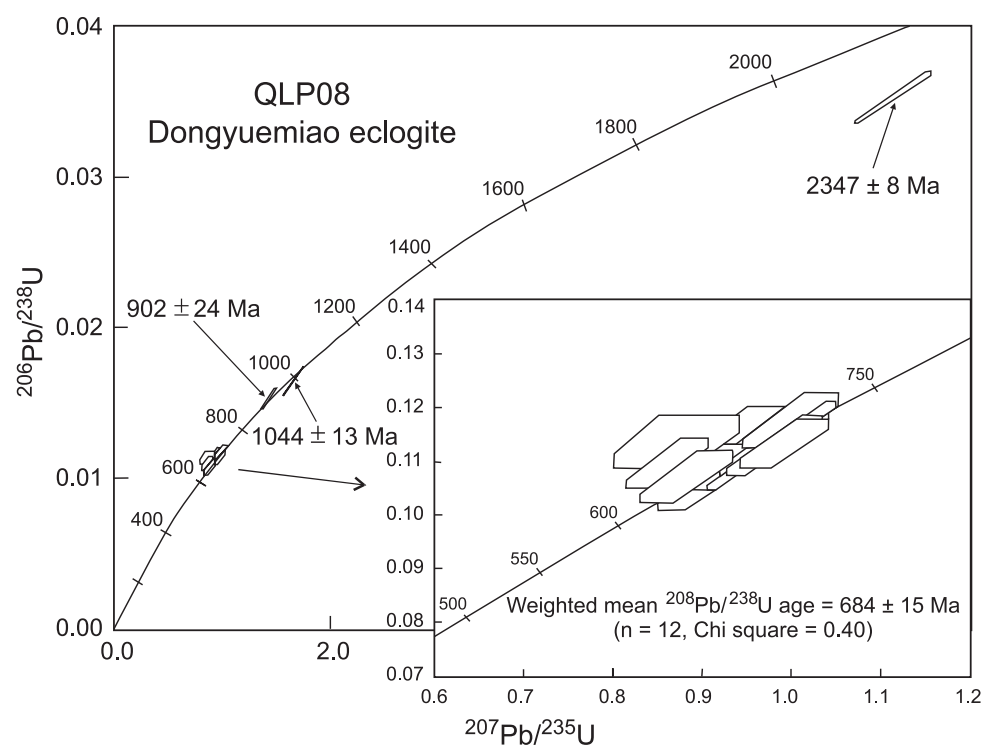

Fig. 7. U-Pb concordia diagram for zircons from the Dongyuemiao eclogite (QLP08). 
Shuanghe area of eastern Dabieshan (Chavagnac et al., 2001). The age is also compatible with the $\mathrm{Nd}$ depleted mantle model ages of a jadeite quartzite ( 2.58 $\mathrm{Ga}$; Liou et al., 1997). Consequently, the presence of a Paleoproterozoic to Late Archean crust in the Yangtze craton is implied.

Zircon may crystallize at low-temperature eclogite facies conditions $\left(\leq 550{ }^{\circ} \mathrm{C}\right)$ in the presence of hydrous fluids (Rubatto et al., 1999; Liermann et al., 2002). However, like in the low- to mediumtemperature eclogites from western Dabieshan, zircon overgrowth is generally too small to be analyzed by the ion microprobe. Still worse, some grains did not even grow under the peak metamorphic conditions. Thus, application of the SHRIMP technique to rocks metamorphosed at lower temperatures $\left(<600{ }^{\circ} \mathrm{C}\right)$ has been scanty and it remains largely unexplored (Rubatto et al., 1998). Some zircons from sample QJH01 are big enough for analysis although they contain numerous mineral inclusions. The metamorphic age of $229 \pm 12$ Ma obtained for this sample is identical to that obtained for the
Xuanhuadian eclogite in the Hong'an unit $(232 \pm 10$ Ma; Sun et al., 2002). These data suggest that the Huwan unit is an indivisible part of the Triassic Dabie-Sulu HP/UHP terrane. Although the metamorphic age of sample QLP08 was not revealed by the ion microprobe analysis, the phengite-whole rock (WR) or phengite-clinopyroxene $\mathrm{Rb}-\mathrm{Sr}$ tie lines give ages of $224 \pm 5$ and $226 \pm 5 \mathrm{Ma}$, respectively (Table 3). A three-point isochron yields $225 \pm 34 \mathrm{Ma}$ (initial ${ }^{87} \mathrm{Sr} /{ }^{86} \mathrm{Sr}=0.7043 \pm 0.0008$ ) for this sample (Fig. 8a). Furthermore, a lower intercept age of $229 \pm 22 \mathrm{Ma}$ was obtained for a granitic gneiss (sample P333) by conventional TIMS U-Pb analysis on five zircons (Table 4; Fig. 9). This sample was collected about $8 \mathrm{~km}$ southwest of sample QLP08. In view of the above consistent Triassic ages, we propose that the peak HP metamorphism in western Dabieshan occurred at ca. 230 Ma, comparable with the peak UHP metamorphic age of eastern Dabieshan (Hacker et al., 1998, 2000; Li et al., 2000; Chavagnac et al., 2001; Ayers et al., 2002; Jahn et al., 2003a).

Table 3

$\mathrm{Rb}-\mathrm{Sr}$ isotopic compositions of eclogites and a blueschist from western Dabieshan

\begin{tabular}{|c|c|c|c|c|c|c|c|}
\hline Whole rock and mineral & $\mathrm{Rb}(\mathrm{ppm})$ & $\mathrm{Sr}(\mathrm{ppm})$ & ${ }^{87} \mathrm{Rb} /{ }^{86} \mathrm{Sr}$ & ${ }^{87} \mathrm{Sr} /{ }^{86} \mathrm{Sr}$ & $\pm 2 \sigma \mathrm{m}$ & Phg- $\phi$ (Ma) & $\begin{array}{l}\text { Initial ratio } \\
\text { (metamorphic) }\end{array}$ \\
\hline \multicolumn{8}{|c|}{ Dongyuemiao eclogite (QLP08) } \\
\hline WR & 10.05 & 142.5 & 0.204 & 0.705001 & 6 & $224 \pm 5$ & $0.70435 \pm 4$ \\
\hline Grt & 1.48 & 2.08 & 2.059 & 0.706312 & 8 & & \\
\hline Cpx & 1.21 & 120.3 & 0.029 & 0.704314 & 6 & $226 \pm 5$ & $0.70422 \pm 4$ \\
\hline Phg & 305.1 & 160.4 & 5.505 & 0.721882 & 7 & & \\
\hline \multicolumn{8}{|l|}{ Tianpu eclogite (TP03) } \\
\hline WR & 11.02 & 297.0 & 0.107 & 0.705051 & 7 & $212 \pm 4$ & $0.70473 \pm 4$ \\
\hline Grt & 0.84 & 34.50 & 0.070 & 0.704817 & 7 & $213 \pm 4$ & $0.70460 \pm 4$ \\
\hline Cpx & 0.98 & 46.08 & 0.061 & 0.704950 & 7 & $211 \pm 4$ & $0.70477 \pm 4$ \\
\hline Phg & 197.7 & 77.42 & 7.391 & 0.726989 & 7 & & \\
\hline Zo & 0.65 & 3819 & 0.0005 & 0.704733 & 6 & $213 \pm 4$ & $0.70473 \pm 4$ \\
\hline \multicolumn{8}{|c|}{ Duanjiagang blueschist (BJ01-101) } \\
\hline WR & 69.31 & 216.3 & 0.9262 & 0.711825 & 7 & $217 \pm 4$ & $0.70897 \pm 9$ \\
\hline Grt & 4.25 & 168.6 & 0.0728 & 0.709485 & 7 & $216 \pm 4$ & $0.70926 \pm 4$ \\
\hline Gln & 5.75 & 133.9 & 0.1239 & 0.709683 & 7 & $216 \pm 4$ & $0.70930 \pm 4$ \\
\hline Phg & 234.8 & 19.11 & 35.888 & 0.819695 & 8 & & \\
\hline $\mathrm{Pl}$ & 4.40 & 96.40 & 0.1317 & 0.706872 & 7 & $222 \pm 4$ & $0.70646 \pm 4$ \\
\hline Kfs & 3.71 & 81.48 & 0.1314 & 0.707138 & 7 & $221 \pm 4$ & $0.70672 \pm 4$ \\
\hline
\end{tabular}

$\mathrm{Rb}-\mathrm{Sr}$ isotope analyses were performed at Géosciences Rennes. Analytical procedures were described by Chavagnac and Jahn (1996) and Jahn et al. (2003a). Phengite-phase two-point tie lines or "isochrons" are calculated using ISOPLOT/Ex 2.01 (Ludwig, 1999). Error input in isochron calculation is $2 \%$ for ${ }^{87} \mathrm{Rb} /{ }^{86} \mathrm{Sr}$, and $0.005 \%$ for ${ }^{87} \mathrm{Sr} /{ }^{86} \mathrm{Sr}$. Decay constant $\left(\lambda^{87} \mathrm{Rb}\right)$ used is $0.0142 \mathrm{Ga}^{-1}$. Mineral abbreviations are as in Table 1 except: $\mathrm{Kfs}=\mathrm{K}$-feldspar, WR=whole rock. 

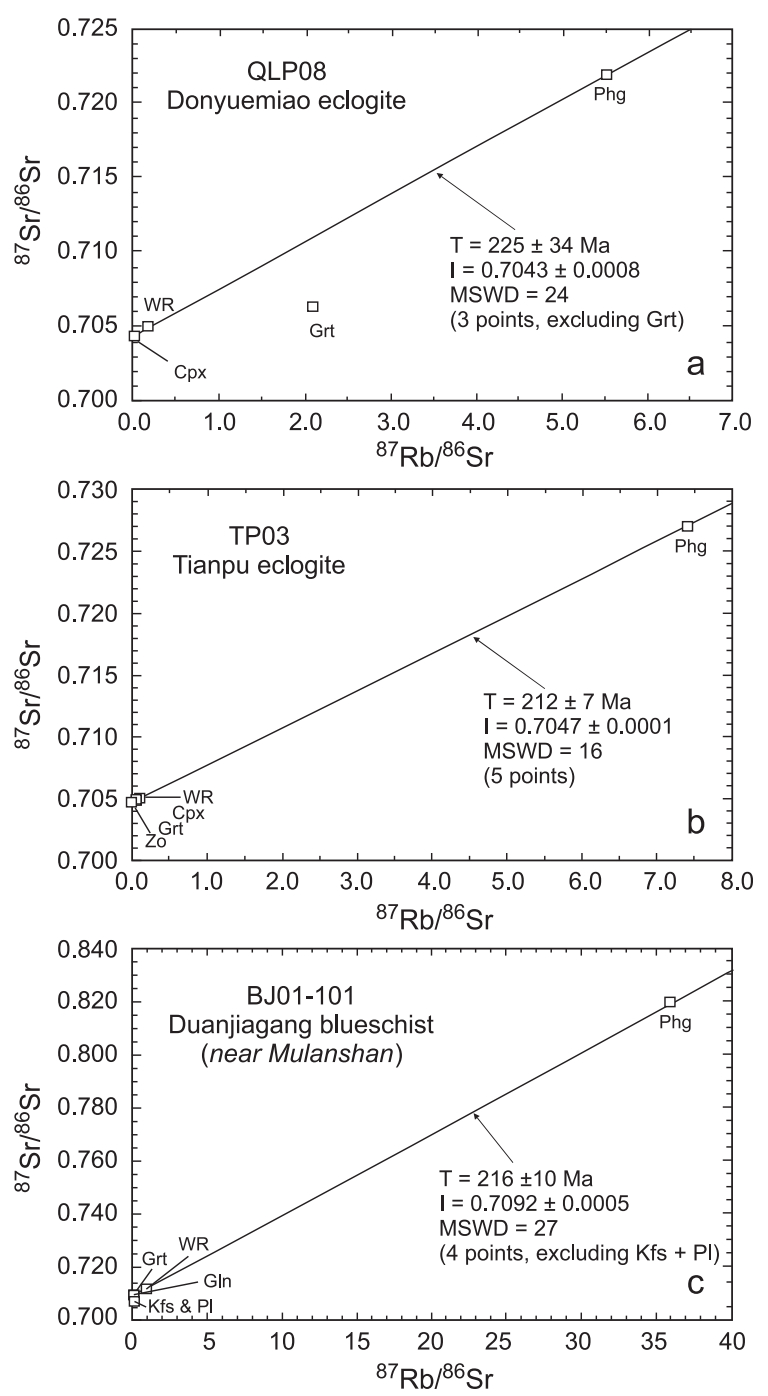

Fig. 8. Phengite-based $\mathrm{Rb}-\mathrm{Sr}$ isochron diagrams for (a) the Dongyuemiao eclogite (QLP08), (b) the Tianpu eclogite (TP03) and (c) the Duanjiagang blueschist, near Mulanshan (BJ01-101).

The U-Pb age of $213 \pm 5 \mathrm{Ma}$ obtained for the Tianpu UHP eclogite is younger than other HP eclogites, but perfectly coincides with the phengitebased $\mathrm{Rb}-\mathrm{Sr}$ isochron age of $212 \pm 7 \mathrm{Ma}$ (initial $\left.{ }^{87} \mathrm{Sr} /{ }^{86} \mathrm{Sr}=0.7047 \pm 0.0001\right)$ obtained for the same sample (Table 3; Fig. 8b). In fact, ages of ca. 213 Ma have been reported for many UHP rocks from eastern Dabieshan using a variety of chronometric methods, including monazite $\mathrm{U}-\mathrm{Pb}$ analysis (Ayers et al., 2002), mineral-WR Sm-Nd and Rb-Sr isochrons (Chavagnac and Jahn, 1996; Li et al., 2000) and phengite ${ }^{40} \mathrm{Ar} /{ }^{39} \mathrm{Ar}$ dating (Hacker et al., 2000; Chavagnac et al., 2001). Most workers attributed this age to representing a regional retrograde metamorphism corresponding to the amphibolite facies (e.g., Hacker et al., 1998, 2000; Li et al., 2000; Ayers et al., 2002). Based on the textures, concordance of zircon analyses and measured age distributions, Ayers et al. (2002) interpreted the monazite ages of 209-213 Ma as the time for new growth and/or recrystallization as a result of pervasive fluid infiltration during retrograde overprinting. The nearly uniform age and cloudy texture of zircons from the Tianpu eclogite seem to suggest a complete post-UHP recrystallization, rather than new growth. The absence of Neoproterozoic protolith age in this rock lends support to this interpretation. Recrystallization of zircons in the presence of fluid could lead to reset of the $\mathrm{U}-\mathrm{Pb}$ isotopic chronometer of protolith zircons (Pidgeon, 1992; Pidgeon et al., 1998). The consistency between $\mathrm{U}-\mathrm{Pb}$ and $\mathrm{Rb}-\mathrm{Sr}$ ages implies that the recrystallization of zircons in the Tianpu eclogite took place at relatively low temperature, probably close to the $\mathrm{Rb}-$ Sr blocking temperatures of phengite. In any case, the age of ca. $213 \mathrm{Ma}$ is likely to represent a cooling age of the UHP rocks during exhumation. This conclusion may be supported by a phengite ${ }^{40} \mathrm{Ar} /{ }^{39} \mathrm{Ar}$ age of $212 \pm 1$ Ma obtained for a tectonic gneiss (sample P306) near the southern margin of the Xinxian UHP unit (our unpublished data).

The Xiongdian eclogite from the Huwan HP unit (Fig. 1) has been the subject of geochronological controversy for many years. U-Pb zircon ages of 400 to $424 \mathrm{Ma}$ for the eclogite were regarded as the time of an Early Paleozoic HP metamorphism by Jian et al. (1997, 2000). These ages were later reinterpreted as the inheritance of the eclogite precursor by $\mathrm{Li}$ et al. (2001) and Sun et al. (2002). Sun et al. (2002) obtained a much younger age of ca. $310 \mathrm{Ma}$, and considered it as the time of Carboniferous HP metamorphic event, to be distinguished from the Triassic HP/UHP event. On the other hand, phengite ${ }^{40} \mathrm{Ar} /{ }^{39} \mathrm{Ar}$ ages of $350-430 \mathrm{Ma}$ were obtained for the same eclogite by $\mathrm{Xu}$ et al. (2000), but the ages were questioned by Sun et al. (2002) for possible presence of inherited argon. Indeed, we also obtained early Paleozoic amphibole and phengite ${ }^{40} \mathrm{Ar} /{ }^{39} \mathrm{Ar}$ ages $(450 \pm 6,410 \pm 9$ and $400 \pm 2 \mathrm{Ma})$ for three eclogites from the Huwan and Hong'an units (our unpublished 


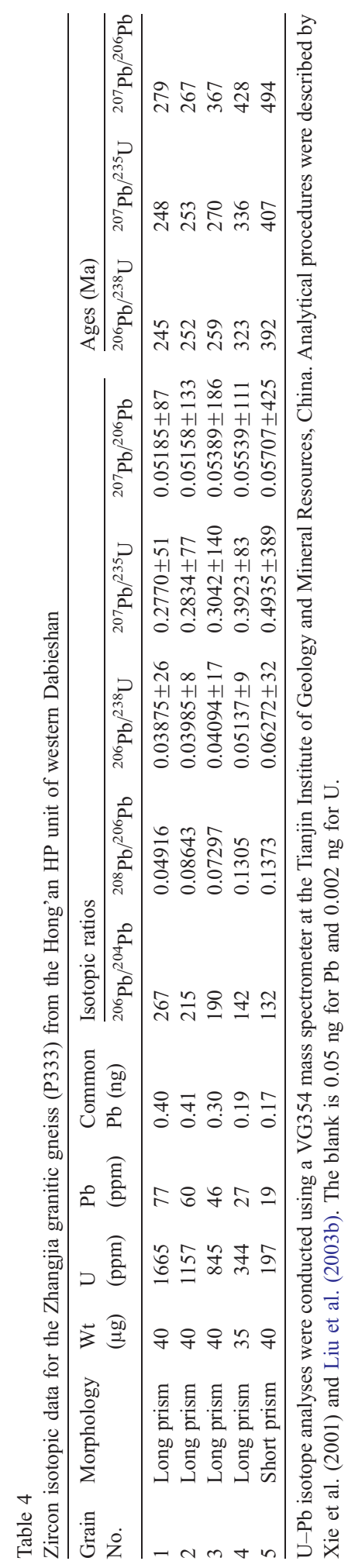

data). Particularly for the Qianjinhepeng eclogite (sample QJH01), the amphibole ${ }^{40} \mathrm{Ar} /{ }^{39} \mathrm{Ar}$ age of $450 \pm 6 \mathrm{Ma}$ is clearly older than the metamorphic zircon age of $229 \pm 12 \mathrm{Ma}$. This probably indicates a widespread existence of excess argon in eclogitic minerals. By contrast, Gao et al. (2002) obtained a U$\mathrm{Pb}$ zircon age of $216 \pm 4 \mathrm{Ma}$ for the Xiongdian eclogite. This agrees with Triassic HP/UHP metamorphic ages obtained for other eclogites, hence providing important argument against a Carboniferous HP metamorphism as advocated by some workers ( $\mathrm{Li}$ et al., 2001; Sun et al., 2002). In our opinion, the nature of ca. $310 \mathrm{Ma}$ event still remains obscure. Even if this event truly results in eclogite facies metamorphism, such Carboniferous eclogites are only confined to the northwestern corner. Its occurrence should not be extrapolated to the whole Huwan unit. The assumption of a double-suture tectonic setting (Sun et al., 2002), a Carboniferous and a Triassic, lying on the two sides of the Huwan unit cannot be supported with the presently available age data. In fact, the westconverging arcuate structures in the west of the Xinxian unit do not support this hypothesis either.

\subsection{Tectonic implications}

In this study, we have consistently obtained Neoproterozoic protolith ages $(680-730 \mathrm{Ma})$ and a Triassic metamorphic age (ca. $230 \mathrm{Ma}$ ) for the Huwan and Hong'an units. Meanwhile, the thermobarometric study indicates that the peak metamorphic P-T conditions of eclogites from the two units are also comparable, $550-570{ }^{\circ} \mathrm{C}$ and $21 \mathrm{kbar}$ for the Huwan unit, and $530-560{ }^{\circ} \mathrm{C}$ and $20-22 \mathrm{kbar}$ for the Hong' an unit (Liu et al., 2004). This demonstrates that the strongly deformed and shortened Huwan unit is equivalent to the less deformed Hong'an unit, and both of them belong to the same HP slice overlying a UHP slice (=main part of the Xinxian unit; see P-P' profile of Fig. 1). The similar volcanosedimentary sequences of the two units lend support to this conclusion. This HP slice is separated from the Xinxian structural dome in the eastern part, but they become a single unit to the west in light of the closed arcuate fault. The structural pattern testifies the hypothesis that the architecture of the Dabie orogen is a huge antiform (Hacker et al., 1998, 2000; Eide and Liou, 2000), although such antiform had been 


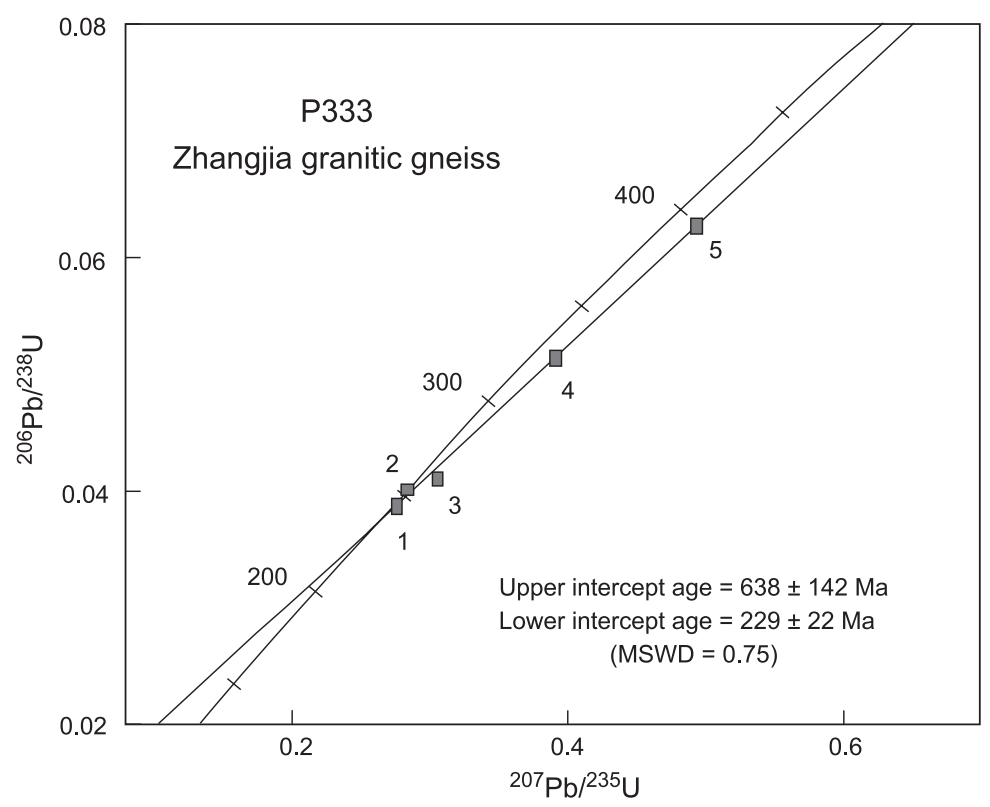

Fig. 9. U-Pb concordia diagram for zircons from the Zhangjia granitic gneiss (P333).

transformed into a structural dome during the Cretaceous subhorizontal extension (Faure et al., 1999, 2003). Based on the mode of occurrence, the Mulanshan blueschist-greenschist unit belongs to a low-temperature and high-pressure (LT/HP) slice overlying the HP slice.

With regard to the Balifan unit, its tectonic affinity has never been clearly defined. It was put into the Huwan HP unit as the Huwan shear zone (e.g., Hacker et al., 2000; Webb et al., 2001), or into the Nanwan unit as a Devonian flysch sequence (e.g., $\mathrm{Xu}$ et al., 2000; Sun et al., 2002). However, the occurrence of widespread mylonites and numerous metagabbro blocks, the absence of eclogites and the presence of faults on the north and south sides are in favor of the Balifan complex as a distinctive unit. This unit was only overprinted by the Triassic epidote-amphibolite facies metamorphism (Liu et al., 2004). Hacker et al. (2000) reported a Neoproterozoic age of $\sim 733$ Ma for a quartzofeldspathic orthogneiss (sample DS337) from this unit. We obtained an emplacement age of $582 \pm 11 \mathrm{Ma}$ for the Sujiahe metagabbro in this study. In addition, $\mathrm{Li}$ et al. (2001) reported $\mathrm{Sm}-\mathrm{Nd}$ and $\mathrm{Rb}-$ Sr whole rock isochron ages of $446 \pm 23$ and $444 \pm 31$ Ma for metavolcanic rocks from the western part of the Balifan unit (i.e., Dingyuan formation). The metavolcanic rocks were inferred to be a component of a magmatic arc in the southern margin of the SinoKorean craton. However, taking into account the two age groups (1000-700 and 500-440 Ma) obtained from detrital zircons from the Foziling flysch sequence of eastern Dabieshan (equivalent to the Nanwan unit; Chen et al., 2003), such magmatic arc should be developed in the northern margin of the Yangtze craton, as Ratschbacher et al. (2003) also suggested. Therefore, we infer that the Balifan tectonic mélange and Nanwan flysch units represent the basement and cover accretionary complexes, respectively, sliced from the Yangtze craton during continental subduction. If correct, the northern boundary fault of the Nanwan unit, as shown in Fig. 1, should be the Triassic suture between the SinoKorean and Yangtze cratons, as Hacker et al. (1998) suggested.

In view of the above analysis, a Triassic spatial restoration of different tectonic units in western Dabieshan is illustrated in Fig. 10. From the north (hanging wall) to the south (footwall), the tectonic units represent successively the suture, cover accretionary complex, basement accretionary complex, LT/ HP slice, HP slice and UHP slice. Phengite ${ }^{40} \mathrm{Ar} /{ }^{39} \mathrm{Ar}$ cooling age obtained from a granitic mylonite (sample 
(a) Subduction and uplift (240-210 Ma)

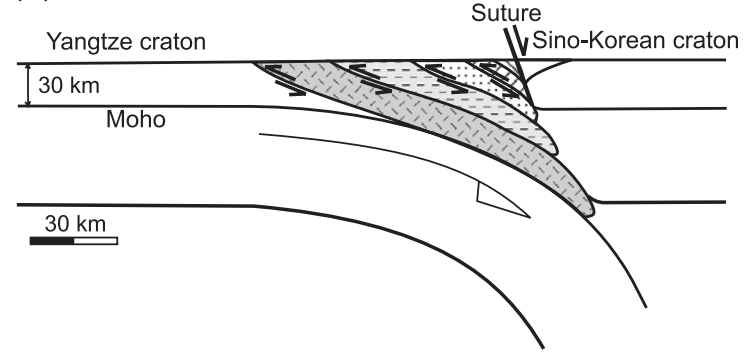

(b) Extrusion and thrusting (210-170 Ma)

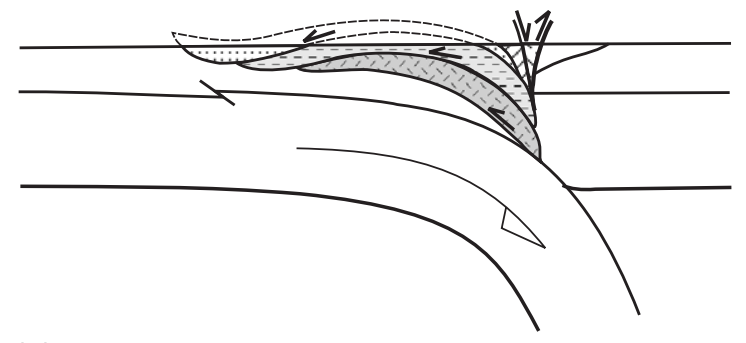

(c) Doming and intrusion (130-110 Ma)

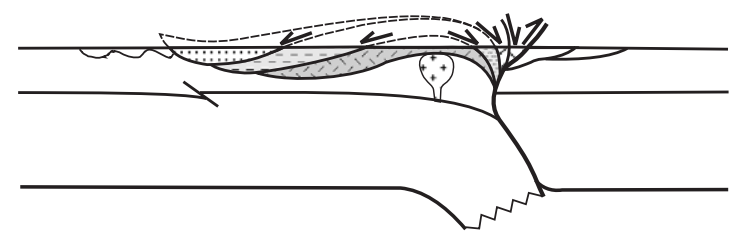

IIV Cover accretionary complex (Nanwan flysch unit)

VIד Basement accretionary complex (Balifan tectonic melange unit)

HP slice (Huwan and Hong'an HP units + Xinxian HP eclogite)

UHP slice (Xinxian UHP unit)

LT/HP slice (Mulanshan blueschist-greenschist unit)

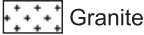

Fig. 10. A tectonic model for the exhumation of the subducted slices in western Dabieshan. (a) Successive subduction of underlying slices with nearly concomitant uplift of overlying slices during the Triassic (240-210 Ma) collision between the Sino-Korean and Yangtze cratons. (b) Extrusion and thrusting of subducted slices onto the Yangtze craton during the Jurassic (210-170 Ma) convergence. (c) Doming and magmatic intrusion during the Cretaceous (130-110 Ma) extension. The Moho offsets below the suture and the southern boundary of the Dabie orogen were inferred from the geophysical surveys (Yuan et al., 2003; Dong et al., 2004).

BLF01) in the basement accretionary complex (Balifan unit) is $241 \pm 2 \mathrm{Ma}$ (our unpublished data), which is similar to that (about $235 \mathrm{Ma}$ ) reported by Webb et al. (1999) and Hacker et al. (2000). By contrast, most phengite ${ }^{40} \mathrm{Ar} /{ }^{39} \mathrm{Ar}$ ages from the HP and LT/HP slices (Huwan, Hong'an and Mulanshan units) range from about 230 to $210 \mathrm{Ma}$ (Eide et al., 1994; Webb et al., 1999, 2001; Hacker et al., 2000). We also obtained a phengite ${ }^{40} \mathrm{Ar} /{ }^{39} \mathrm{Ar}$ age of $231 \pm 1$ Ma for a quartzofeldspathic mylonite (sample P531) from the southernmost margin of the Mulanshan unit (our unpublished data). Meanwhile, a WR-garnet-glaucophane-phengite $\mathrm{Rb}-\mathrm{Sr}$ isochron age of $216 \pm 10 \mathrm{Ma}$ was obtained for a quartzofeldspathic blueschist (sample BJ01-101) from this unit (Table 3; Fig. 8c). It appears that all the $\mathrm{U}-\mathrm{Pb}, \mathrm{Rb}-\mathrm{Sr}$ and ${ }^{40} \mathrm{Ar} /{ }^{39} \mathrm{Ar}$ dating results suggest a cooling age of ca. $210 \mathrm{Ma}$ for the UHP slice (main part of the Xinxian unit). The implication from the age data above is that the exhumation to the crustal levels of different units probably took place diachronously and lasted for about $30 \mathrm{Ma}$. That is, the exhumation of shallow accretionary complex predates the later exhumation of the UHP slice by about $30 \mathrm{Ma}$. The diachronous exhumation could be explained by a successive subduction-exhumation model. As shown in Fig. 10, during the 240-210 Ma interval, the broken slices of the subducting Yangtze slab reached at different depths and the successive subduction of underlying slices was accompanied by nearly concomitant uplift of overlying slices (Fig. 10a). Subsequently (201-170 $\mathrm{Ma}$ ), the southward thrusting by a combination of coeval normal sense shear from beneath the SinoKorean craton led to an upward extrusion of the subducted slices onto the Yangtze craton (Fig. 10b). During the Cretaceous extension (130-110 Ma), doming and extensive plutonism resulted in the present structure and distribution of tectonic units, and the old thrust are reworked as ductile normal faults (Fig. 10c; Faure et al., 1999; Hacker et al., 2000; Yuan et al., 2003).

\section{Conclusions}

The present age study leads to the following conclusions:

(1) The principal eclogites from the Huwan HP unit (Huwan shear zone) were produced by the Triassic (ca. $230 \mathrm{Ma}$ ) HP metamorphism from the Neoproterozoic protoliths. The Huwan unit 
is therefore an integral part of the entire Triassic Dabie-Sulu HP/UHP terrane, but not a part of the Carboniferous eclogite belt as proposed by some authors (Li et al., 2001; Sun et al., 2002). The geological meaning of a few Carboniferous zircon ages for eclogites from the northwestern corner of western Dabieshan remains obscure.

(2) The Balifan tectonic mélange and Nanwan flysch units located to the north of the HP/ UHP complexes represent the basement and cover accretionary complexes, respectively. They were sliced from the Yangtze craton during the Triassic continental subduction. The northern boundary fault of the Nanwan unit is inferred to be the Triassic suture between the Sino-Korean and Yangtze cratons.

(3) The Huwan and Hong'an HP units belong to the same HP slice overlying the UHP slice. The exhumation of the subducted slab components, from the hanging wall accretionary complex to the footwall UHP slice, took place diachronously and lasted about 30 Ma during the Triassic. This could be explained by a successive subduction of underlying slices in combination with a nearly concomitant exhumation of overlying slices.

\section{Acknowledgements}

We sincerely thank Ping Jian for technical assistance in the zircon $\mathrm{U}-\mathrm{Pb}$ analyses on SHRIMP II in Beijing. Nicole Morin and Joël Macé of Géosciences Rennes kindly performed the $\mathrm{Rb}-\mathrm{Sr}$ isotopic analyses reported herein. Critical reviews by B. Hacker and M. Faure and editorial comments by J.-P. Burg substantially improved the manuscript. The research of the senior author was supported by the Major State Basic Research Development Program of China (TG1999075505), Geological Investigation Project of China Geological Survey (200013000169) and Free Research Project of MLR (2002406). He was further supported by the National Science Council of Taiwan for the final preparation of the manuscript. Bor-ming Jahn acknowledges the financial support of the NSCTaiwan (NSC91-2811-M-002-012, NSC92-2811-M002-056 and NSC92-2116-M-002-024).

\section{References}

Ames, L., Tilton, G.R., Zhou, G., 1993. Timing of collision of the Sino-Korean and Yangtze cratons: U-Pb Zircon dating of coesite-bearing eclogites. Geology 21, 339-342.

Ames, L., Zhou, G., Xiong, B., 1996. Geochronology and isotopic character of ultrahigh-pressure metamorphism with implications for collision of the Sino-Korean and Yangtze Cratons, central China. Tectonics 15, 472-489.

Ayers, J.C., Dunkle, S., Gao, S., Miller, C.F., 2002. Constraints on timing of peak and retrograde metamorphism in the Dabie Shan ultrahigh-pressure metamorphic belt, east-central China, using $\mathrm{U}-\mathrm{Th}-\mathrm{Pb}$ dating of zircon and monazite. Chem. Geol. 186, 315-331.

Black, L.P., Kamo, S.L., Allen, C.M., Aleinikoff, J.N., Davis, D.W., Korsch, R.J., Foudoulis, C., 2003. TEMORA 1: a new standard for Phanerozoic U-Pb geochronology. Chem. Geol. 200, 155-170.

Burton, K.W., Kohn, M.G., Cohen, A.S., O’Nions, R.K., 1995. The relative diffusion of $\mathrm{Pb}, \mathrm{Nd}, \mathrm{Sr}$ and $\mathrm{O}$ in garnet. Earth Planet. Sci. Lett. 133, 199-211.

Carswell, D.A., Harley, S.L., 1990. Mineral barometry and thermometry. In: Carswell, D.A. (Ed.), Eclogite Facies Rocks. Blackie, New York, pp. 83-110.

Carswell, D.A., O’Brien, P.J., Wilson, R.N., Zhai, M., 1997. Thermobarometry of phengite-bearing eclogites in the Dabie Mountains of central China. J. Metamorph. Geol. 15, 239-252.

Chavagnac, V., Jahn, B.-m., 1996. Coesite-bearing eclogites from the Bixiling Complex, Dabie Mountains, China: Sm-Nd ages, geochemical characteristics and tectonic implications. Chem. Geol. 133, 29-51.

Chavagnac, V., Jahn, B.-m., Villa, I.M., Whitehouse, M.J., Liu, D., 2001. Multichronometric evidence for an in situ origin of the ultrahigh-pressure metamorphic terrane of Dabieshan, China. J. Geol. 109, 633-646.

Chen, F., Guo, J., Jiang, L., Siebel, W., Cong, B., Satir, M., 2003. Provenance of the Beihuaiyang lower-grade metamorphic zone of the Dabie ultrahigh-pressure collisional orogen, China: evidence from zircon ages. J. Asian Earth Sci. 120, 131-148.

Compston, W., Williams, I.S., Kirschvink, J.L., Zhang, Z., Ma, G., 1992. Zircon U-Pb ages for the Early Cambrian time-scale. J. Geol. Soc. London 149, 171-184.

Cui, W., Wang, X., 1995. Eclogites of southern Henan and northern Hubei Provinces, central China. Isl. Arc 4, 347-361.

Cumming, G.L., Richards, G.R., 1975. Ore lead isotope ratios in a continuously changing Earth. Earth Planet. Sci. Lett. 28, $155-171$.

Dong, S., Gao, R., Cong, B., Zhao, Z., Liu, X., Li, S., Li, Q., Huang, D., 2004. Crustal structure of the southern Dabie ultrahigh-pressure orogen and Yangtze foreland from deep seismic reflection profiling. Terra Nova (in press).

Eide, E.A., Liou, J.G., 2000. High-pressure blueschists and eclogites in Hong'an: a frame work for addressing the evolution of high-and ultrahigh-pressure rocks in central China. Lithos 52, $1-22$.

Eide, E.A., Mcwilliams, M.O., Liou, J.G., $1994 .{ }^{40} \mathrm{Ar} /{ }^{39} \mathrm{Ar}$ geochronology and exhumation of high-pressure to ultrahigh- 
pressure metamorphic rocks in east central China. Geology 22, $601-604$.

Faure, M., Lin, W., Shu, L., Sun, Y., Schärer, U., 1999. Tectonics of the Dabieshan (eastern China) and possible exhumation mechanism of ultra high-pressure rocks. Terra Nova 11, 251-258.

Faure, M., Lin, W., Schärer, U., Shu, L., Sun, Y., Arnaud, N., 2003. Continental subduction and exhumation of the UHP rocks. Structural and geochronological insights from the Dabieshan (East China). Lithos 70, 213-241.

Fu, B., Zheng, Y.F., Touret, J.L.R., 2002. Petrological, isotopic and fluid inclusion studies of eclogites from Sujiahe, NW Dabie Shan (China). Chem. Geol. 187, 107-128.

Gao, S., Qiu, Y., Ling, W., McNaughton, N.J., Zhang, B., Zhang, G., Zhang, Z., Zhong, Z., Suo, S., 2002. SHRIMP single zircon $\mathrm{U}-\mathrm{Pb}$ geochronology of eclogites from Yingshan and Xiongdian. Earth Sci. 27, 558-564. (in Chinese with English Abstract).

Gebauer, D., Schertl, H.-P., Brix, M., Schreyer, W., 1997. 35 Ma old ultrahigh-pressure metamorphism and evidence for very rapid exhumation in the Dora Maira Massif, Western Alps. Lithos 41, 5-24.

Günther, M., Jagoutz, E., 1997. Isotope disequilibria (Sm/Nd, Rb/ $\mathrm{Sr}$ ) between minerals of coarse grained, low temperature garnet peridotites from Kimberley floors, Southern Africa. In: Meyer, H.O.A., Leonardos, O.H. (Eds.), Proceedings of 5th International Kimberlite Conference 1, CPRM Spec. Publ. 1A (Brasilia), pp. 354-365.

Hacker, B.R., Wang, X., Eide, E.A., Ratschbacher, L., 1996. The Qinling-Dabie ultra-high-pressure collisional orogen. In: Yin, A., Harrison, M.T. (Eds.), The Tectonic Evolution of Asia. Cambridge University Press, Cambridge, pp. 345-370.

Hacker, B.R., Ratschbacher, L., Webb, L.E., Ireland, T., Walker, D., Dong, S., 1998. Zircon ages constrain the architecture of the ultrahigh-pressure Qinling-Dabie orogen, China. Earth Planet. Sci. Lett. 161, 215-230.

Hacker, B.R., Ratschbacher, L., Webb, L.E., McWilliams, M.O., Ireland, T., Calvert, A., Dong, S., Wenk, H.-R., Chateigner, D., 2000. Exhumation of ultrahigh-pressure continental crust in east central China: Late Triassic-Early Jurassic tectonic unroofing. J. Geophys. Res. 105, 13339-13364.

Jaeger, E., Niggli, E., Wenk, E., 1967. Rb-Sr Altersbestimmungen an Glimmern der Zentralalpen. Beitr. Geol. Kt. Schweiz 134 (67 pp.).

Jahn, B.-m., Liu, X., 2002. Age (s) of the Hong'an block and the question of 400 Ma UHP metamorphic event. International Workshop on Geophysics and Structure Geology of UHPM Terranes (Extended Abstract). Beijing, China, pp. 68-70.

Jahn, B.-m., Fan, Q., Yang, J.-J., Henin, O., 2003a. Petrogenesis of the Maowu pyroxenite-eclogite body from the UHP metamorphic terrane of Dabieshan: chemical and isotopic constraints. Lithos 70, 243-267.

Jahn, B.-m., Rumble, D., Liou, J.G., 2003b. Geochemistry and isotope tracer study of UHPM rocks (Chapter 12). In: Carswell, D.A., Compagnoni, R. (Eds.), Ultra-high Pressure Metamorphism, EMU Notes in Mineralogy, vol. 5, pp. 365-414.
Jian, P., Yang, W., Li, Z., 1997. Isotopic geochronological evidence for the Caledonian Xiongdian eclogite in the western Dabie Mountains, China. Acta Geol. Sin. 71, 133-141. (in Chinese with English Abstract).

Jian, P., Liu, D., Yang, W., Williams, I.S., 2000. Petrographical study of zircons and SHRIMP dating of the Caledonian Xiongdian eclogite, northwestern Dabie Mountains. Acta Geol. Sin. 74, 259-264. (in Chinese with English Abstract).

Li, S., Xiao, Y., Liu, D., Chen, Y., Ge, N., Zhang, Z., Sun, S.-s., Cong, B., Zhang, R., Hart, S.R., Wang, S., 1993. Collision of the North China and Yangtze Blocks and formation of coesite-bearing eclogites: timing and processes. Chem. Geol. 109, 89-111.

Li, S., Wang, S., Chen, Y., Zhou, H., Zhang, Z., Liu, D., Qiu, J., 1994. Excess argon in phengite from eclogite: evidence from dating of eclogite minerals by $\mathrm{Sm}-\mathrm{Nd}, \mathrm{Rb}-\mathrm{Sr}$ and ${ }^{40} \mathrm{Ar} /{ }^{39} \mathrm{Ar}$ methods. Chem. Geol. 112, 343-350.

Li, S., Jagoutz, E., Chen, Y., Li, Q., 2000. Sm-Nd and Rb-Sr isotopic chronology and cooling history of ultrahigh pressure metamorphic rocks and their country rocks at Shuanghe in the Dabie Mountains, central China. Geochim. Cosmochim. Acta 64, 1077-1093.

Li, S., Huang, F., Nie, Y., Han, W., Long, C., Li, H., Zhang, S., Zhang, Z., 2001. Geochemical and geochronological constraints on the suture location between the North and South China Blocks in the Dabie orogen, central China. Phys. Chem. Earth, A 26, 655-672.

Li, X.H., Li, Z.X., Ge, W., Zhou, H., Li, W., Liu, Y., Wingate, M.T.D., 2003a. Neoproterozoic granitoids in south China: crustal melting above a mantle plume at ca. $825 \mathrm{Ma}$ ? Precambrian Res. 122, 45-83.

Li, Z.X., Li, X.H., Kinny, P.D., Wang, J., Zhang, S., Zhou, H., 2003b. Geochronology of Neoproterozoic syn-rift magmatism in the Yangtze Craton, South China and correlations with other continents: evidence for a mantle superplume that broke up Rodinia. Precambrian Res. 122, 85-109.

Liati, A., Gebauer, D., 1999. Constraining the prograde and retrograde $\mathrm{P}-\mathrm{T}-\mathrm{t}$ path of Eocene HP rocks by SHRIMP dating of different zircon domains: inferred rates of heating, burial, cooling and exhumation for central Rhodope, northern Greece. Contrib. Mineral. Petrol. 135, 340-354.

Liermann, H.-P., Isachsen, C., Altenberger, U., Oberhänsli, R., 2002. Behavior of zircon during high-pressure, low-temperature metamorphism: case study from the Internal Unit of the Sesia Zone (Western Italian Alps). Eur. J. Mineral. 14, 61-71.

Liou, J.G., Zhang, R.Y., Wang, X., Eide, E.A., Ernst, W.G., Maruyama, S., 1996. Metamorphism and tectonics of highpressure and ultra-high-pressure belts in the Dabie-Sulu region, China. In: Yin, A., Harrison, M.T. (Eds.), The Tectonic Evolution of Asia. Cambridge University Press, Cambridge, pp. $300-344$.

Liou, J.G., Zhang, R.Y., Jahn, B.-m., 1997. Petrology, geochemistry and isotope data on a ultrahigh-pressure jadeite quartzite from Shuanghe, Dabie Mountains, East-central China. Lithos 41, 59-78.

Liou, J.G., Zhang, R.Y., Katayama, I., Maruyama, S., 2002. Global distribution and petrotectonic character- 
izations of UHPM terranes. In: Parkinson, C.D., Katayama, I., Liou, J.G., Maruyama, S. (Eds.), The Diamond-Bearing Kokchetav Massif, Kazakhstan. Universal Academy Press, Tokyo, pp. 15-35.

Liu, D., Jian, P., Zhang, Q., Cao, H., 2003a. SHRIMP dating of adakites in the Tulingkai oophiolite, Inner Mongonia: evidence for the early Paleozoic subduction. Acta Geol. Sin. 77, 317-327. (in Chinese with English Abstract).

Liu, X., Jahn, B.-m., Dong, S., Li, H., Oberhänsli, R., 2003b. Neoproterozoic granitoid did not record ultrahigh-pressure metamorphism from the southern Dabieshan of China. J. Geol. $111,719-732$.

Liu, X., Wei, C., Li, S., Dong, S., Liu, J., 2004. Thermobaric structure of a traverse across western Dabieshan: implications for collision tectonics between the Sino-Korean and Yangtze cratons. J. Metamorph. Geol. 22, 361-379.

Ludwig, K.R., 1999. Isoplot/Ex (v. 2.01)—a geochronological toolkit for Microsoft Excel. Berkeley Geochronology Center, Special Publication, No. 1a. 49 pp.

Maruyama, S., Tabata, H., Nutman, A.P., Morikawa, T., Liou, J.G., 1998. SHRIMP U-Pb geochronology of ultrahigh-pressure metamorphic rocks of the Dabie Mountains, central China. Continental Dynamics 3, 72-85.

Mezger, K., Essene, E.J., Halliday, A.N., 1992. Closure temperatures of the $\mathrm{Sm}-\mathrm{Nd}$ system in metamorphic garnets. Earth Planet. Sci. Lett. 113, 397-409.

Okay, A.I., Sengör, A.M.C., Satir, M., 1993. Tectonics of an ultrahigh-pressure metamorphic terrane: the Dabie Shan/Tongbai Shan orogen, China. Tectonics 12, 1320-1334.

Paulsson, O., Austrheim, H., 2003. A geochronological and geochemical study of rocks from Gjelsvikfjella, Dronning Maud Land, Antarctica-implications for Mesoproterozoic correlations and assembly of Gondwana. Precambrian Res. $125,113-138$.

Pidgeon, R.T., 1992. Recrystallisation of oscillatory zoned zircon: some geochronological and petrological implications. Contrib. Mineral. Petrol. 110, 463-472.

Pidgeon, R.T., Nemchin, A.A., Hitchen, G.J., 1998. Internal structures of zircons from Archaean granites from the Darling Range batholith: implications for zircon stability and the interpretation of zircon $\mathrm{U}-\mathrm{Pb}$ ages. Contrib. Mineral. Petrol. $132,288-299$.

Powell, R., 1985. Regression diagnostics and robust regression in geothermometer/geobarometer calibration: the garnet-clinopyroxene geothermometer revisited. J. Metamorph. Geol. 3, $231-243$.

Powell, R., Holland, T., Worley, B., 1998. Calculating phase diagrams involving solid solutions via non-linear equations, with examples using Thermocalc. J. Metamorph. Geol. 16, $577-588$.

Ratschbacher, L., Hacker, B.R., Calvert, A., Webb, L.E., Grimmer, J.C., Mcwilliams, M.O., Ireland, T., Dong, S., Hu, J., 2003. Tectonics of the Qinling (Central China): tectonostratigraphy, geochronology, and deformation history. Tectonophysics 366, $1-53$.

Rowley, D.B., Xue, F., Tucker, R.D., Peng, Z.X., Baker, J., Davis, A., 1997. Ages of ultrahigh pressure metamor- phism and protolith orthogneisses from the eastern Dabie Shan: U/Pb zircon geochronology. Earth Planet. Sci. Lett. 151, $191-203$.

Rubatto, D., Gebauer, D., 2000. Use of cathodoluminescence for U$\mathrm{Pb}$ zircon dating by ion microprobe; some examples from the Western Alps. In: Pagel, M., Barbin, V., Blanc, P., Ohnenstetter, D. (Eds.), Cathodoluminescence in Geosciences. Springer, Berlin, pp. 373-400.

Rubatto, D., Gebauer, D., Fanning, M., 1998. Jurassic formation and Eocene subduction of the Zermatt-Saas-Fee ophiolites: implications for the geodynamic evolution of the Central and Western Alps. Contrib. Mineral. Petrol. 132, 269-287.

Rubatto, D., Gebauer, D., Compagnoni, R., 1999. Dating of eclogite-facies zircons: the age of Alpine metamorphism in the Sesia-Lanzo Zone (Western Alps). Earth Planet. Sci. Lett. 167, $141-158$.

Sun, W., Williams, I.S., Li, S., 2002. Carboniferous and Triassic eclogites in the western Dabie Mountains, east-central China: evidence for protracted convergence of the North and South China Blocks. J. Metamorph. Geol. 20, 873-886.

Thöni, M., 2002. Sm-Nd isotope systematics in garnet from different lithologies (Eastern Alps): age results, and an evaluation of potential problems for garnet $\mathrm{Sm}-\mathrm{Nd}$ chronometry. Chem. Geol. 185, 255-281.

Tilton, G.R, Schreyer, W., Schertl, H.-P., 1991. Pb-Sr-Nd isotopic behavior of deeply subducted crustal rocks from the Dora Maira massif, wetern Alps, Italy: II. What is the age of the ultrahighpressure metamorphism? Contrib. Mineral. Petrol. 108, 22-33.

Webb, L.E., Hacker, B.R., Ratschbacher, L., McWilliams, M.O., Dong, S., 1999. Thermochronologic constraints on deformation and cooling history of high- and ultrahigh-pressure rocks in the Qinling-Dabie orogen, eastern China. Tectonics 18, 621-638.

Webb, L.E., Ratschbacher, L., Hacker, B.R., Dong, S., 2001. Kinematics of exhumation of high-and ultrahigh-pressure rocks in the Hong'an and Tongbai Shan of the Qinling-Dabie collisional orogen, eastern China. In: Hendrix, M.S., Davis, G.A. (Eds.), Paleozoic and Mesozoic Tectonic Evolution of Central Asia: From Continental Assembly to Intracontinental Deformation, Memoir 194. Geological Society of America, Boulder, Colorado, pp. 231-245.

Williams, I.S., Claesson, S., 1987. Isotopic evidence for the Precambrian provenance and Caledonian metamorphism of high grade paragneisses from the Seve Nappes, Scandinavian Caledonides: II. Ion microprobe zircon U-Th- $\mathrm{Pb}$. Contrib. Mineral. Petrol. 97, 205-217.

Williams, I.S., Buick, I.S., Cartwright, I., 1996. An extended episode of early Mesoproterozoic metamorphic fluid flow in the Reynolds Range, central Australia. J. Metamorph. Geol. 14, 29-47.

Wingate, M.T.D., Campbell, I.H., Compston, W., Gibson, G.M., 1998. Ion microprobe U-Pb ages for Neoproterozoic basaltic magmatism in south-central Australia and implications for the breakup of Rodinia. Precambrian Res. 87, 135-159.

Xie, Z., Chen, J.-F., Zheng, Y.-F., Zhang, X., Li, H.-M., Zhou, T.-X., 2001. Zircon $\mathrm{U}-\mathrm{Pb}$ dating of the metamorphic rocks of different grades from the southern part of the Dabie terrain in China. Phys. Chem. Earth (A) 26, 685-693. 
Xu, B., Grove, M., Wang, C., Zhang, L., Liu, S., 2000. ${ }^{40} \mathrm{Ar} /{ }^{39} \mathrm{Ar}$ thermochronology from the northwestern Dabie Shan: constraints on the evolution of Qinling-Dabie orogenic belt, eastcentral China. Tectonophysics 322, 279-301.

Xue, F., Rowley, D.B., Tucker, R.D., Peng, Z., 1997. U-Pb zircon ages of granitoid rocks in the north Dabie Complex, eastern Dabie Shan, China. J. Geol. 105, 744-753.

Yang, J.S., Wooden, J.L., Wu, C.L., Liu, F.L., Xu, Z.Q., Shi, R.D., Katayama, I., Liou, J.G., Maruyama, S., 2003. SHRIMP U-Pb dating of coesite-bearing zircon from the ultrahigh-pressure metamorphic rocks, Sulu terrane, east China. J. Metamorph. Geol. 21, 551-560.

Yuan, X.-C., Klemperer, S.L., Teng, W.-B., Liu, L.-X., Chetwin, E., 2003. Crustal structure and exhumation of the Dabie Shan ultrahigh-pressure orogen, eastern China, from seismic reflection profiling. Geology 31, 435-438.
Zhang, R.Y., Liou, J.G., 1994. Coesite-bearing eclogite in Henan Province, central China: detailed petrography, glaucophane stability and P-T path. Eur. J. Mineral. 6, 217-233.

Zhong, Z., Suo, S., You, Z., 1999. Regional-scale extensional tectonic pattern of ultrahigh-P and high-P metamorphic belts from the Dabie massif, China. Inter. Geol. Rev. 41, 1033-1041.

Zhong, Z., Suo, S., You, Z., Zhang, H., Zhou, H., 2001. Major constituents of the Dabie collisional orogenic belt and partial melting in the ultrahigh-pressure unit. Inter. Geol. Rev. 43, $226-236$.

Zhou, B., Hensen, B.J., 1995. Inherited Sm/Nd isotope components preserved in monazite inclusions within garnets in leucogneiss from East Antarctica and implications for closure temperature studies. Chem. Geol. 121, 317-326. 